\title{
The Robustness of CAPM-A Computational Approach*
}

\author{
P. Jean-Jacques Herings ${ }^{\dagger}$ \\ Felix Kubler ${ }^{\ddagger}$ \\ Department of Economics \\ University of Maastricht \\ Department of Economics \\ Stanford University
}

January 21,2000

\begin{abstract}
In this paper we argue that in realistically calibrated two period general equilibrium models with incomplete markets CAPM-pricing provides a good benchmark for equilibrium prices even when agents are not mean-variance optimizers and returns are not normally distributed. We numerically approximate equilibria for a variety of different specifications for preferences, endowments and dividends and compare the equilibrium prices and portfolio-holdings to the predictions of CAPM. While we show that CAPM cannot hold exactly for the chosen specification, it turns out that pricing-errors are extremely small. Furthermore, two-fund separation holds approximately.
\end{abstract}

JEL codes: C61, C62, C63, C68, D52, D58, G11, G12.

Keywords: asset pricing, general equilibrium, incomplete markets, computational methods.

${ }^{*}$ The research of Jean-Jacques Herings has been made possible by a fellowship of the Royal Netherlands Academy of Arts and Sciences and a grant of the Netherlands Organization for Scientific Research (NWO). While this paper was being written this author enjoyed the generous hospitality of the Cowles Foundation for Research in Economics at Yale University, of CORE at Université Catholique de Louvain, and of CentER at Tilburg University. Felix Kubler gratefully acknowledges the financial support of the Cowles Foundation in the form of an Anderson dissertation fellowship.

${ }^{\dagger}$ P.J.J. Herings, Department of Economics, University of Maastricht, P.O. Box 616, 6200 MD Maastricht, The Netherlands. E-mail: P.Herings@algec.unimaas.nl

${ }_{\ddagger}^{\ddagger}$ F. Kubler, Department of Economics, Stanford University, Stanford, California 94305-6072, USA. 


\section{INTRODUCTION}

The CAPITAl ASSET PRICing MOdel (CAPM) of Sharpe (1964) and Lintner (1965) predicts that equilibrium returns of assets are a linear function of their market $\beta$ (the slope in the regression of a security's return on the market's return). This intuitively appealing result has long shaped the way practitioners think about average returns and risk. While the model fares poorly in explaining observed cross-sectional stock returns (see for example Fama and French (1992)) it remains one of the central building blocks in financial economics.

One of the reasons for this is that the CAPM provides a good theoretical starting point for the examination of asset prices. Geanakoplos and Shubik (1990) show that CAPM can be viewed as a special case of the general equilibrium model with incomplete asset markets (the GEI-model). Oh (1996), Willen (1997) and others have shown that the central conclusions of CAPM, the pricing formula, holds true under completely general dividends and endowments as long as all agents have mean-variance utility functions.

However, without mean-variance preferences one has to make very strong assumptions on the distribution of asset pay-offs in order to derive the conclusions of $\mathrm{CAPM}^{1}$. Berk (1997) shows that joint restrictions on utility functions and asset returns cannot lead to more realistic assumptions. He shows that if one assumes that agents have von-NeumannMorgenstern utility functions, quadratic utility is necessary for the CAPM-pricing formula to hold. Since quadratic utility is an unattractive assumption, it is an important question whether CAPM-pricing provides a benchmark for the cross section of security prices in a model with more general preferences, endowments and asset returns. Empirical contradictions of CAPM might be explained by the fact that some agents are not mean-variance optimizers and that many securities have returns that are far from elliptical.

In this paper we show that independently of mean-variance preferences or normal returns, the CAPM pricing formula often provides a very good prediction for actual equilibrium returns. It is clear, of course, that it is always possible to construct economies where equilibrium asset prices are arbitrarily far from those predicted by CAPM. We do not provide a theoretical explanation for the documented phenomenon but instead compute hundreds of examples which illustrate it (see Judd (1997) for a general discussion which favors this approach to economic theory) - we approximate equilibria numerically (using the algorithm developed in Herings and Kubler (2000)) and compare the prices and portfolio-holdings predicted by CAPM to the actual equilibrium prices and equilibrium portfolio-holdings. In all cases we consider, CAPM pricing is an excellent benchmark for the equilibrium prices. These results are robust with respect to variations in preferences, endowments and dividends.

In order to show that the CAPM pricing formula provides a good approximation to asset returns in realistically calibrated models, one first has to clarify what one means by 'realistically calibrated'. We follow the macroeconomic literature and we choose first

\footnotetext{
${ }^{1}$ Ross (1978) provides a complete characterization of the class of separating distribution
} 
and second moments of endowments and dividends to roughly match annual US data and preferences to exhibit relative risk aversion below 10 and nondecreasing absolute risk aversion (see e.g. Mehra and Prescott (1985)) $)^{2}$.

More importantly one has to argue that the computed examples are not sensitive to the exact specification of the model but that they reflect some general property of asset prices. We assume that there are three agents and 32,768 states of nature and we examine the robustness of CAPM with respect to 600 different specifications of preferences and endowments: We first assume that endowments and dividends are log-normally distributed and consider the following 3 specifications for preferences.

- All three agents have constant absolute risk aversion (CARA) utility functions.

- All agents' utility functions exhibit constant relative risk aversion (CRRA).

- Agents' utility-functions exhibit loss aversion as in Benartzi and Thaler (1995).

For each case we randomly generate 100 economies which differ with respect to agents' (heterogeneous) degrees of risk aversion.

In the next three cases we fix preferences and vary distributions of dividends and endowments. We assume that all agents have CRRA utility functions and consider the following distributions for assets and endowments.

- Endowments and dividends are drawn from a uniform distribution, We randomly generate 100 economies with differ with respect to the support of the uniform distributions.

- Endowments and dividends are determined by two factors and an idiosyncratic shock each of which are drawn from a log-normal distribution. We randomly generate 100 economies with differ with respect to the factor-loads.

- Endowments and dividends are drawn from a log-normal distribution and there is an option on one of the stocks. We randomly generate 100 economies which differ with respect to the strike-price of the option.

For all 600 economies under consideration we compare the computed return on individual stocks to the return predicted by the CAPM-pricing formula. We find that in all 600 cases the average mean squared pricing errors (for returns) across stocks lie below 0.04 percent. The average error across all simulations is in the order of magnitude of 0.005 percent. In addition to predicting asset returns, the CAPM also predicts that all agents' equilibrium portfolio-holdings will consist of the riskless bond and a mutual fund of risky assets. It is possible that CAPM-pricing is very accurate, but two-fund separation does not

\footnotetext{
${ }^{2}$ Note that just as in Mehra and Prescott this calibration is very unrealistic with respect to the market risk-premium. This fact might give a first indication of why CAPM-pricing does so well in our framework.
} 
apply. Nevertheless, in the computed examples two-fund separation holds almost exactly in the equilibria we compute.

The paper is organized as follows. In Section 2 we give a short introduction to the model and collect several general results on CAPM in a general equilibrium setting. Section 3 gives an example of a realistically calibrated economy with non-elliptical returns and CRRA preferences for which CAPM-pricing provides an almost perfect prediction. In Sections 4 and 5 we examine the robustness of this phenomenon. In Section 4 we vary the parameters of risk aversion for the CRRA case, we consider CARA utility functions, and we examine utility functions displaying loss aversion. In Section 5 we fix preferences to exhibit constant relative risk aversion and we examine the robustness of CAPM with respect to dividenddistributions. In Section 6 we speculate about possible explanations and conclude the paper.

\section{The Two-Period Finance Economy}

The finance version of the GEI-model describes an economy over two periods of time, $t=0,1$, with uncertainty over the state of nature resolving in period $t=1$. We describe the model, introduce the necessary notation and discuss the CAPM. For a thorough description of the GEI-model see for example Magill and Quinzii (1996).

\subsection{The MODEL}

There are $S+1$ states in the economy; at time $t=0$ the economy is in state $s=0$, at time $t=1$ one state of nature $s$ out of $S$ possible states of nature realizes. In each state $s=0, \ldots, S$, there is a single nondurable consumption good.

There are $H$ agents, indexed by $h=1, \ldots, H$, that participate in the economy. Each agent $h$ is characterized by initial endowments (the initial income stream) $e^{h}=\left(e_{0}^{h}, e_{1}^{h}, \ldots, e_{S}^{h}\right)^{\top} \in$ $\operatorname{int}\left(X^{h}\right)^{3}$ and his preferences over consumption bundles (income streams available for consumption) $c^{h}=\left(c_{0}^{h}, c_{1}^{h}, \ldots, c_{S}^{h}\right)^{\top} \in X^{h}$. Here $X^{h}$ is a closed subset of $\mathbb{R}^{S+1}$ that satisfies $\left\{x^{h}\right\}+\mathbb{R}_{+}^{S+1} \subset X^{h}$ for all $x^{h} \in X^{h}$. In most applications $X^{h}$ will be equal to $\mathbb{R}_{+}^{S+1}$ or $\mathbb{R}^{S+1}$. To distinguish between first-period consumption and the random second period consumption, we define $\widetilde{x}=\left(x_{1}, \ldots, x_{S}\right)^{\top}$ for any vector $x=\left(x_{0}, x_{1}, \ldots, x_{S}\right)^{\top}$. Aggregate endowments (aggregate incomes) are $e=\sum_{h=1}^{H} e^{h}$. Each agents' preferences are represented by a utility function $u^{h}: X^{h} \rightarrow \mathbb{R}$ satisfying standard assumptions; $u^{h}$ is strictly quasi-concave and continuous. Moreover, the set $X^{h}\left(e^{h}\right)=\left\{x^{h} \in X^{h} \mid u^{h}\left(x^{h}\right) \geq u^{h}\left(e^{h}\right)\right\}$ is assumed to be bounded from below, a property automatically satisfied when $X^{h}$ is bounded from below. In the applications of Sections $3-5$ we consider economies where all agents have separable utility functions across date-events with identical probabilities, i.e. there exist

\footnotetext{
${ }^{3} \operatorname{int}\left(X^{h}\right)$ denotes the interior of $X^{h}$.
} 
probabilities $\rho_{1}, \ldots, \rho_{S}>0, \sum_{s=1}^{S} \rho_{s}=1$, such that

$$
u^{h}\left(c^{h}\right)=v_{0}^{h}\left(c_{0}^{h}\right)+\delta^{h} \sum_{s=1}^{S} \rho_{s} v_{s}^{h}\left(c_{s}^{h}\right),
$$

where $\delta^{h}>0$ is the discount factor, $X^{h}=\prod_{s=0}^{S} X_{s}^{h}$, where $X_{s}^{h}$ is a subset of $\mathbb{R}$, and $v_{s}^{h}: X_{s}^{h} \rightarrow \mathbb{R}$ is assumed to be strictly increasing and strictly concave. In this case it follows from the properties of $v_{s}^{h}$ that $X^{h}\left(e^{h}\right)$ is bounded from below. When the functions $v_{s}^{h}$ are independent of $s$, we say that agents have von Neumann-Morgenstern utility functions.

There are $J$ assets. Asset $j$ pays dividends at date $t=1$ which we denote by $d^{j} \in \mathbb{R}^{S}$. The price of asset $j$ at time $t=0$ is $q_{j}$. Without loss of generality we assume that the assets are in zero net supply and we collect all assets' dividends in a pay-off matrix

$$
A=\left(d^{1}, \ldots, d^{J}\right) \in \mathbb{R}^{S \times J} .
$$

At time $t=0$ agent $h$ chooses a portfolio-holding $\theta^{h} \in \mathbb{R}^{J}$ which uniquely defines the agents' consumption by $\widetilde{c}^{h}=\widetilde{e}^{h}+A \theta^{h}$ and $c_{0}^{h}=e_{0}^{h}-\theta^{h} \cdot q$. The net demand of agent $h$, $\widetilde{c}^{h}-\tilde{e}^{h}$, belongs to the marketed subspace $\langle A\rangle=\left\{z \in \mathbb{R}^{S} \mid \exists \theta \in \mathbb{R}^{J}, z=A \theta\right\}$.

The exogenous parameters defining a finance economy $\mathcal{E}=\left(\left(X^{h}, u^{h}, e^{h}\right)_{h=1, \ldots, H} ; A\right)$ are agents' consumption sets, utility functions and endowments, and the pay-off matrix. Without loss of generality, we assume throughout that there are no redundant assets, $\operatorname{rank}(A)=J$. If there are redundant assets, it follows from an arbitrage argument that their price is uniquely determined by the price of the other assets. Markets are incomplete when $J<S$. We define asset prices to be arbitrage free if it is not possible to achieve a positive income stream in all states by trading in the available assets. It is well known that a price system $q \in \mathbb{R}^{J}$ precludes arbitrage if and only if there exists a state price vector $\pi \in \mathbb{R}_{++}^{S}$ such that $q=\pi^{\top} A$. We define $Q$ to be the set of arbitrage free asset prices.

Definition 2.1 (Competitive Equilibrium): A competitive equilibrium for an economy $\mathcal{E}$ is a collection of portfolio-holdings $\theta^{*}=\left(\theta^{1 *}, \ldots, \theta^{H *}\right) \in \mathbb{R}^{H J}$ and asset prices $q^{*} \in \mathbb{R}^{J}$ that satisfy the following conditions:

(1) $\theta^{h *} \in \arg \max _{\theta^{h} \in \mathbb{R}^{J}} u^{h}\left(c^{h}\right)$ s.t. $c^{h}=e^{h}+\left(\begin{array}{c}-q^{*^{\top}} \\ A\end{array}\right) \theta^{h}$ and $c^{h} \in X^{h}, \quad h=1, \ldots, H$;

(2) $\sum_{h=1}^{H} \theta^{h *}=0$.

Under an additional assumption of strictly increasing utility functions, existence of an equilibrium follows from the results of Geanakoplos and Polemarchakis (1986).

\subsection{The Capital Asset Pricing Model}

Sharpe (1964) and Lintner (1965) use the portfolio analysis developed by Tobin (1958) and Markowitz (1959) to examine an equilibrium model of financial markets. Under the 
assumption that all agents are mean-variance optimizers they derive a closed-form solution for equilibrium returns, the so-called $\beta$-pricing formula. This formula relates the return of a risky asset to the return of the market portfolio by the covariance of that asset with the market. It is well known that the $\beta$-pricing formula can be derived in the finance GEImodel, see Geanakoplos and Shubik (1990). To fix notation and to give some intuition for the computational results in Sections $3-5$, we summarize and slightly generalize the findings in the literature - Geanakoplos and Shubik (1990), Magill and Quinzii (1996), Oh (1996), and Willen (1997) - to cover the case with mean-variance preferences, non-marketed endowments and a finite state space in a world with incomplete markets.

We denote by $1_{n}=(1, \ldots, 1)^{\top} \in \mathbb{R}^{n}$ the vector of all ones. The $m$-th unit vector of appropriate dimension is denoted $\iota_{m}$ - the dimension of $\iota_{m}$ is always apparent from the context. Throughout this subsection we assume that there exist objective probabilities $\rho_{s}$, $s=1, \ldots, S$, over the possible states of nature in period 1 . Moreover, asset 1 is a riskless bond, $d^{1}=1_{S}$. For a random variable $x \in \mathbb{R}^{S}$, we define its expected value $\mathrm{E}(x)=$ $\sum_{s=1}^{S} \rho_{s} x_{s}$, for two random variables $x, y \in \mathbb{R}^{S}$, we define the covariance as $\operatorname{cov}(x, y)=$ $\sum_{s=1}^{S} \rho_{s} x_{s} y_{s}-\mathrm{E}(x) \mathrm{E}(y)$. The variance of a random variable $x \in \mathbb{R}^{S}$ is given by $\operatorname{var}(x)=$ $\operatorname{cov}(x, x)$. Finally, we define $x \cdot{ }_{\rho} y=\sum_{s=1}^{S} \rho_{s} x_{s} y_{s}$ for vectors $x, y \in \mathbb{R}^{S}$.

For any competitive equilibrium $\left(\theta^{*}, q^{*}\right)$, there exists a unique state price vector in the marketed subspace $\pi_{A}^{*} \in\langle A\rangle$ such that, for all assets $j q_{j}^{*}=\pi_{A}^{*}{ }_{\rho} d^{j}$. Using the definitions of variance and covariance, this implies

$$
q_{j}^{*}=\mathrm{E}\left(\pi_{A}^{*}\right) \mathrm{E}\left(d^{j}\right)+\operatorname{cov}\left(\pi_{A}^{*}, d^{j}\right) .
$$

We define the return of a portfolio $\theta \in \mathbb{R}^{J}$ with $q^{*} \cdot \theta \neq 0$ by $r_{\theta}=\frac{A \theta}{q^{*} \cdot \theta}$ and we denote the return of the riskless bond by $R^{\mathrm{f}}=\frac{1}{q_{1}^{*}}$. With this we rewrite equation (1) as

$$
q_{j}^{*}=\frac{1}{R^{\mathrm{f}}} \mathrm{E}\left(d^{j}\right)+\operatorname{cov}\left(\pi_{A}^{*}, d^{j}\right) .
$$

We define the pricing portfolio as the unique portfolio $\theta_{A}^{*}$ which solves $A \theta_{A}^{*}=\pi_{A}^{*}$.

Notice that

$$
q^{*} \cdot \theta_{A}^{*}=\pi_{A}^{*} \cdot \rho A \theta_{A}^{*}=\pi_{A}^{*} \cdot \pi_{A}^{*}>0
$$

where $\pi_{A}^{*} \neq 0$ follows from $\mathrm{E}\left(\pi_{A}^{*}\right)=q_{1}^{*}>0$.

Since the return of the pricing portfolio satisfies $r_{\theta_{A}^{*}}=\frac{A \theta_{A}^{*}}{q^{*} \cdot \theta_{A}^{*}}=\frac{\pi_{A}^{*}}{\pi_{A}^{*} \cdot \pi_{A}^{*}}$ we can rewrite equation (1) as

$$
\mathrm{E}\left(r_{\theta}\right)-R^{\mathrm{f}}=\frac{\operatorname{cov}\left(r_{\theta}, r_{\theta_{A}^{*}}\right)}{\operatorname{var}\left(r_{\theta_{A}^{*}}\right)}\left(\mathrm{E}\left(r_{\theta_{A}^{*}}\right)-R^{\mathrm{f}}\right) .
$$

While equation (2) relates the prices of the risky assets and looks similar to the CAPM pricing formula, this formula is rather useless if we have no further information on $\pi_{A}^{*}$. Note that so far all formulas followed simply from the absence of arbitrage. It is well known that under the assumption that one agent $h$ 's utility function is differentiable and that in an 
equilibrium with individual consumption $\left(c^{h *}\right)_{h \in H}$, agent $h$ 's utility maximization problem has an interior solution, $\pi_{A}^{*}$ can be characterized as

$$
\pi_{A}^{*}=\operatorname{proj}_{\langle A\rangle}\left(\frac{\partial_{c_{1}^{h}} u^{h}\left(c^{h *}\right) / \rho_{s}}{\partial_{c_{0}^{h}} u^{h}\left(c^{h *}\right)}, \ldots, \frac{\partial_{c_{S}^{h}} u^{h}\left(c^{h *}\right) / \rho_{s}}{\partial_{c_{0}^{h}} u^{h}\left(c^{h *}\right)}\right),
$$

where $\operatorname{proj}_{\langle A\rangle}$ denotes the projection on $\langle A\rangle$ under the inner product $\rho$.

One possibility to derive an interesting pricing formula is to assume that all preferences just depend on the mean and the variance of consumption,

$$
u^{h}\left(c^{h}\right)=w^{h}\left(c_{0}^{h}, \mathrm{E}\left(\widetilde{c}^{h}\right), \operatorname{var}\left(\widetilde{c}^{h}\right)\right),
$$

where $w^{h}$ is strictly increasing in $c_{0}^{h}$ and in the expected consumption and strictly decreasing in the variance of consumption.

Agent $h$ 's first period endowments can be decomposed into a marketed part and a nonmarketed part, where the latter part lies orthogonal to the marketed subspace under the inner product $\rho$. We write

$$
\widetilde{e}^{h}=\widetilde{e}_{\mathrm{M}}^{h}+\widetilde{e}_{\perp}^{h}
$$

and have by definition $\widetilde{e}_{\perp}^{h} \cdot{ }_{\rho} z=0$ for all $z \in\langle A\rangle$. This decomposition is uniquely determined. We define the marketed endowments $\widetilde{e}_{\mathrm{M}}=\sum_{h=1}^{H} \widetilde{e}_{\mathrm{M}}^{h}$ and the market portfolio $\theta_{\mathrm{M}}$ as the unique portfolio satisfying

$$
A \theta_{\mathrm{M}}=\widetilde{e}_{\mathrm{M}} \text {. }
$$

Note that it may happen that $q^{*} \cdot \theta_{\mathrm{M}}=0$, even when $\widetilde{e} \gg 0 .^{4}$

To simplify matters, we first assume $q^{*} \cdot \theta_{\mathrm{M}} \neq 0$ and then argue that this assumption is not necessary. Given a competitive equilibrium $\left(\theta^{*}, q^{*}\right)$, we define $\beta_{\theta}$ for a portfolio $\theta \in \mathbb{R}^{J}$ by

$$
\beta_{\theta}=\frac{\operatorname{cov}\left(r_{\theta}, r_{\theta_{\mathrm{M}}}\right)}{\operatorname{var}\left(r_{\theta_{\mathrm{M}}}\right)} .
$$

Then we have the following result.

THEOREM 2.3: Under the assumptions that all agents maximize mean-variance utility functions with objective probabilities $\rho, \operatorname{var}\left(\widetilde{e}_{\mathrm{M}}\right)>0$, and there is a riskless bond, each equilibrium $\left(\theta^{*}, q^{*}\right)$ of $\mathcal{E}$ with equilibrium consumption $\left(c^{1 *}, \ldots, c^{H *}\right)$ has the following properties.

1. The CAPM-pricing formula holds; when $q^{*} \cdot \theta_{\mathrm{M}} \neq 0$, then for each $\theta \in \mathbb{R}^{J}$,

$$
E\left(r_{\theta}\right)-R^{\mathrm{f}}=\beta_{\theta}\left(E\left(r_{\theta_{\mathrm{M}}}\right)-R^{\mathrm{f}}\right) .
$$

\footnotetext{
${ }^{4}$ For a vector $x \in \mathbb{R}^{m}$ we use the notation $x \geq 0$ if $x \in \mathbb{R}_{+}^{m}, x>0$ if $x \in \mathbb{R}_{+}^{m} \backslash\{0\}$, and $x \gg 0$ if $x \in \mathbb{R}_{++}^{m}$.
} 
2. Two-fund separation holds; for each agent $h$ there exists $\left(\alpha_{1}^{h}, \alpha_{2}^{h}\right) \in \mathbb{R} \times \mathbb{R}_{+}$, where $\sum_{h=1}^{H} \alpha_{1}^{h}=0$ and $\sum_{h=1}^{H} \alpha_{2}^{h}=1$, such that

$$
\widetilde{c}^{h *}-\widetilde{e}_{\perp}^{h}=\alpha_{1}^{h} 1_{S}+\alpha_{2}^{h} A \theta_{\mathrm{M}}
$$

3. The pricing vector satisfies $\pi_{A}^{*}=\alpha_{1} 1_{S}-\alpha_{2} \widetilde{e}_{\mathrm{M}}$, with $\alpha_{1}>\alpha_{2} \mathrm{E}(\widetilde{e})$ and $\alpha_{2}$ strictly positive.

ProOF. We first show that a pseudo two-fund separation holds in the sense that the agents' consumption bundles can be written as $\widetilde{c}^{h *}=\widetilde{e}_{\perp}^{h}+\widetilde{\alpha}_{1}^{h} 1_{S}+\widetilde{\alpha}_{2}^{h} \pi_{A}^{*}$ for some $\widetilde{\alpha}_{1}^{h}, \widetilde{\alpha}_{2}^{h} \in \mathbb{R}$. Define

$$
\widetilde{c}^{h}=\widetilde{e}_{\perp}^{h}+\operatorname{proj}_{\left\langle 1_{S}, \pi_{A}^{*}\right\rangle}\left(\widetilde{c}^{h *}\right) .
$$

Suppose pseudo two-fund separation does not hold, so $\widetilde{c}^{h} \neq \widetilde{c}^{h *}$. Since $\pi_{A}^{*} \cdot{ }_{\rho}\left(\widetilde{c}^{h *}-\widetilde{c}^{h}\right)=0$, it follows that the portfolios needed to consume $\widetilde{c}^{h *}$ and $\widetilde{c}^{h}$ are as expensive at date 0 . Moreover, $\widetilde{e}_{\perp}^{h} \cdot \rho\left(\widetilde{c}^{h *}-\widetilde{c}^{h}\right)=0$ and $1_{S} \cdot{ }_{\rho}\left(\widetilde{c}^{h *}-\widetilde{c}^{h}\right)=0$, so it follows that $\mathrm{E}\left(\widetilde{c}^{h *}-\widetilde{c}^{h}\right)=0$ and $\operatorname{cov}\left(\widetilde{c}^{h *}-\widetilde{c}^{h}, \widetilde{c}^{h}\right)=0$. Therefore, $\mathrm{E}\left(\widetilde{c}^{h *}\right)=\mathrm{E}\left(\widetilde{c}^{h}\right)$ and $\operatorname{var}\left(\widetilde{c}^{h *}\right)>\operatorname{var}\left(\widetilde{c}^{h}\right)$, giving a contradiction to the optimality of $\widetilde{c}^{h *}$ at prices $q^{*}$. We obtain pseudo two-fund separation.

Since in equilibrium $\widetilde{e}_{\mathrm{M}}=\sum_{h=1}^{H}\left(\widetilde{c}^{h *}-\widetilde{e}_{\perp}^{h}\right)$, the two-fund separation property implies that $\widetilde{e}_{\mathrm{M}} \in\left\langle 1_{S}, \pi_{A}^{*}\right\rangle$. The assumption $\operatorname{var}\left(\widetilde{e}_{\mathrm{M}}\right)>0$ implies that $\widetilde{e}_{\mathrm{M}}$ is not collinear to $1_{S}$ and it holds that $\pi_{A}^{*}=\alpha_{1} 1_{S}-\alpha_{2} \widetilde{e}_{\mathrm{M}}$ for some numbers $\alpha_{1}, \alpha_{2}$. Two-fund separation follows immediately, $\widetilde{c}^{h *}-\widetilde{e}_{\perp}^{h}=\alpha_{1}^{h} 1_{S}+\alpha_{2}^{h} A \theta_{\mathrm{M}}$ for some numbers $\alpha_{1}^{h}, \alpha_{2}^{h}$.

Since $\widetilde{e}_{\mathrm{M}}=\sum_{h=1}^{H} \alpha_{1}^{h} 1_{S}+\sum_{h=1}^{H} \alpha_{2}^{h} \widetilde{e}_{\mathrm{M}}$ and $\operatorname{var}\left(\widetilde{e}_{\mathrm{M}}\right)>0$, we have $\sum_{h=1}^{H} \alpha_{1}^{h}=0$ and $\sum_{h=1}^{H} \alpha_{2}^{h}=1$. Consider a consumption bundle $c_{-}^{h}$ that results from using the income that is invested in the market portfolio to buy the riskless bond, so $\widetilde{c}_{-}^{h}=\widetilde{e}_{\perp}^{h}+\alpha_{1}^{h} 1_{S}+\alpha_{2}^{h}\left(\pi_{A}^{*} \cdot \rho\right.$ $\left.A \theta_{\mathrm{M}} / q_{1}^{*}\right) 1_{S}$. The portfolios needed to consume $\widetilde{c}^{h *}$ and $\widetilde{c}_{-}^{h}$ are as expensive since $\pi_{A}^{*} \cdot{ }_{\rho}\left(\widetilde{c}^{h *}-\right.$ $\left.\widetilde{c}_{-}^{h}\right)=0$. Since $\operatorname{var}\left(\widetilde{c}_{-}^{h}\right) \leq \operatorname{var}\left(\widetilde{c}^{h *}\right)$ and $u^{h}\left(c_{-}^{h}\right) \leq u^{h}\left(c^{h *}\right)$, it holds that $\mathrm{E}\left(\widetilde{c}_{-}^{h}\right)-\mathrm{E}\left(\widetilde{c}^{h *}\right)=$ $-\left(\alpha_{2}^{h} \alpha_{2} / q_{1}^{*}\right) \operatorname{var}\left(\widetilde{e}_{\mathrm{M}}\right) \leq 0$, where we use that $\pi_{A}^{*} \cdot{ }_{\rho} A \theta_{\mathrm{M}}=q_{1}^{*} \mathrm{E}\left(\widetilde{e}_{\mathrm{M}}\right)-\alpha_{2} \operatorname{var}\left(\widetilde{e}_{\mathrm{M}}\right)$. The preceding inequalities are strict inequalities when $\alpha_{2}^{h}>0$, which is the case for at least one agent. Then it follows that $\alpha_{2}>0$ and $\alpha_{2}^{h} \geq 0, h=1, \ldots, H$. Since $0<q_{1}^{*}=\mathrm{E}\left(\pi_{A}^{*}\right)=\alpha_{1}-$ $\alpha_{2} \mathrm{E}\left(\widetilde{e}_{\mathrm{M}}\right)$, and $1_{S} \cdot{ }_{\rho} \widetilde{e}_{\perp}=0$, so $\mathrm{E}\left(\widetilde{e}_{\mathrm{M}}\right)=\mathrm{E}(\widetilde{e})$, it holds that $\alpha_{1}>\alpha_{2} \mathrm{E}(\widetilde{e})$.

The CAPM pricing formula is obtained by substituting $\theta_{A}^{*}=\alpha_{1} \iota_{1}-\alpha_{2} \theta_{\mathrm{M}}$ in equation (2). From

$$
\frac{\operatorname{cov}\left(r_{\theta}, r_{\theta_{A}^{*}}\right)}{\operatorname{var}\left(r_{\theta_{A}^{*}}\right)}=-\frac{q^{*} \cdot \theta_{A}^{*}}{\alpha_{2} q^{*} \cdot \theta_{\mathrm{M}}} \frac{\operatorname{cov}\left(r_{\theta}, r_{\theta_{\mathrm{M}}}\right)}{\operatorname{var}\left(r_{\theta_{\mathrm{M}}}\right)}
$$

and

$$
\mathrm{E}\left(r_{\theta_{A}^{*}}\right)-R^{\mathrm{f}}=\frac{\alpha_{1}-\alpha_{2} \mathrm{E}\left(A \theta_{\mathrm{M}}\right)}{q^{*} \cdot \theta_{A}^{*}}-R^{\mathrm{f}},
$$


it follows that

$$
\mathrm{E}\left(r_{\theta}\right)-R^{\mathrm{f}}=\beta_{\theta}\left(\frac{\alpha_{1}-\alpha_{2} \mathrm{E}\left(A \theta_{\mathrm{M}}\right)}{-\alpha_{2} q^{*} \cdot \theta_{\mathrm{M}}}+\frac{R^{\mathrm{f}}\left(\alpha_{1} q_{1}^{*}-\alpha_{2} q^{*} \cdot \theta_{\mathrm{M}}\right)}{\alpha_{2} q^{*} \cdot \theta_{\mathrm{M}}}\right)=\beta_{\theta}\left(E\left(r_{\theta_{\mathrm{M}}}\right)-R^{\mathrm{f}}\right) .
$$

Q.E.D.

We assume in the theorem that $\operatorname{var}\left(\widetilde{e}_{\mathrm{M}}\right)>0$. The theorem also holds true for the degenerate case where $\widetilde{e}_{\mathrm{M}}$ is collinear to $1_{S}$, but since the proof of this simple fact is rather tedious it is omitted.

Note that for the case where the endowments are spanned, i.e. where $e_{\perp}^{h}=0$ for all $h$, the pricing formula reduces to the standard CAPM-formula (see Magill and Quinzii (1996)).

It might be sensible to define the market portfolio somewhat differently as a portfolio of risky assets only. This clarifies the concept of two-fund separation, since then one fund consists of risky assets only. In this case define $\widehat{\theta}_{\mathrm{M}}=\left(0, \theta_{\mathrm{M}, 2}, \ldots, \theta_{\mathrm{M}, J}\right)$. If we define $\widehat{\beta}_{\theta}=\operatorname{cov}\left(r_{\theta}, r_{\widehat{\theta}_{\mathrm{M}}}\right) / \operatorname{var}\left(r_{\widehat{\theta}_{\mathrm{M}}}\right)$ it turns out that the pricing formula still holds. After some substitutions, one obtains

$$
\mathrm{E}\left(r_{\theta}\right)-R^{\mathrm{f}}=\widehat{\beta}_{\theta}\left(\mathrm{E}\left(r_{\widehat{\theta}_{\mathrm{M}}}\right)-R^{\mathrm{f}}\right) .
$$

Even more generally, define the market portfolio $\widetilde{\theta}_{\mathrm{M}}$ as an arbitrary combination of a portfolio consisting of the riskless asset only and the portfolio $\widehat{\theta}_{\mathrm{M}}$, so

$$
\widetilde{\theta}_{\mathrm{M}}=\gamma_{1} \iota_{1}+\gamma_{2} \widehat{\theta}_{\mathrm{M}}
$$

where $\gamma_{2} \neq 0$. Then it holds that $\theta_{A}^{*}=\widetilde{\alpha}_{1} \iota_{1}-\widetilde{\alpha}_{2} \widetilde{\theta}_{\mathrm{M}}$, where $\widetilde{\alpha}_{2} \neq 0$. If we define $\widetilde{\beta}_{\theta}=$ $\operatorname{cov}\left(r_{\theta}, r_{\widetilde{\theta}_{\mathrm{M}}}\right) / \operatorname{var}\left(r_{\widetilde{\theta}_{\mathrm{M}}}\right)$, then

$$
\mathrm{E}\left(r_{\theta}\right)-R^{\mathrm{f}}=\widetilde{\beta}_{\theta}\left(\mathrm{E}\left(r_{\widetilde{\theta}_{\mathrm{M}}}\right)-R^{\mathrm{f}}\right) .
$$

The proof is identical to the one of Theorem 2.3, when $\alpha_{1}, \alpha_{2}$, and $\theta_{\mathrm{M}}$ are substituted by $\widetilde{\alpha}_{1}, \widetilde{\alpha}_{2}$, and $\widetilde{\theta}_{\mathrm{M}}$. This result also offers a way out when $q^{*} \cdot \theta_{\mathrm{M}}=0$. One may simply use $\widetilde{\theta}_{\mathrm{M}}=\theta_{\mathrm{M}}+\varepsilon \iota_{1}$ with $\varepsilon>0$ to derive the pricing formula. Indeed, $q^{*} \cdot \widetilde{\theta}_{\mathrm{M}}=\varepsilon q_{1}^{*}>0$.

The version of two-fund separation we consider in Theorem 2.3 is slightly more general than the usual one, where it is assumed that initial the initial income stream $e^{h}$ of every agent is marketed. As a consequence one obtains the formula

$$
\widetilde{c}^{h *}=\alpha_{1}^{h} 1_{S}+\alpha_{2}^{h} \widetilde{e}
$$

when endowments are marketed. In the more general case considered in Theorem 2.3, the final income stream consumed by each agent consists not only of the returns of a linear combination of the riskless bond and the market portfolio, but also of the undiversifiable non-marketed individual part of the initial income stream, $\widetilde{e}_{\perp}^{h}$.

Finally, note that the concept of marketed endowments is not needed to define the pricing vector. Since $\widetilde{e}_{\perp}$ is orthogonal to $\langle A\rangle$, the pricing vector can also be defined by 
$\tilde{\pi}_{A}^{*}=\alpha_{1} 1_{S}-\alpha_{2} \widetilde{e}$. Of course it no longer holds that $\tilde{\pi}_{A}^{*} \in\langle A\rangle$. Moreover, income streams not in $\langle A\rangle$ are typically priced differently by $\tilde{\pi}_{A}^{*}$ than by $\pi_{A}^{*}$.

As we have discussed in the introduction, Theorem 2.3 can only be obtained when one is willing to make very restrictive assumptions. As Magill and Quinzii (1996) put it when commenting on representative agent models and the CAPM: "As we indicated above these models are interesting since they lead to clearcut results which have strong intuitive appeal. However the restrictive nature of the hypothesis made could cast doubt on the generality of the results." The important question we want to address is how much actual equilibrium prices and actual portfolio-holdings in a general setting will differ from the predictions of CAPM.

\section{CAPM Without Mean-Variance Preferences}

The assumption that all agents maximize a quadratic utility function is unattractive because it implies increasing absolute risk aversion. A more realistic assumption, and one commonly made in macroeconomics and finance, is that agents' preferences exhibit constant relative risk aversion. It is clear, however, that with these preferences agents' will care about higher moments and that therefore a mean-variance analysis is not valid. The following example shows that a mean-variance utility function does not even serve as a good approximation of a constant relative risk aversion utility function.

EXAMPLE 3.1: Consider an agent with utility function $u^{h}\left(c^{h}\right)=\sum_{s=1}^{3} \rho_{s} v^{h}\left(c_{s}^{h}\right)$, where $\rho_{s}=1 / 3, s=1,2,3$, and $v^{h}\left(c_{s}^{h}\right)=-1 / 3\left(c_{s}^{h}\right)^{-3}$, which corresponds to a utility function with constant relative risk aversion equal to 4 . For simplicity we assume that the household has no income at $t=0$ and does not derive utility from consumption in that period. Consider the consumption of two income streams, $(0.8,0.8,1.4)$ and $(0.6,1.2,1.2)$, that have the same mean and variance. Any mean-variance utility function should therefore consider both income streams as being equally good. When an agent has a constant relative risk aversion utility function, the second income stream is less preferred, as the income in the first state is $40 \%$ lower than average income, whereas the income at the bad states of the first income stream are only $20 \%$ below average income, $u^{h}(0.8,0.8,1.4)=-0.475$ and $u^{h}(0.6,1.2,1.2)=-0.643$. Even if for the second income profile, income is increased by $10 \%$ in every state, we get $u^{h}(0.66,1.32,1.32)=-0.483$, so it would still be inferior to the income stream $(0.8,0.8,1.4)$. This phenomenon becomes even more severe when two income streams with the same, higher variance are compared or when a more risk averse agent is considered.

A standard way to calibrate equilibrium models under uncertainty is to assume that there are several uncorrelated shocks and to choose the magnitude of the shocks to match aggregate first and second moments. From now on we examine an economy with three 
heterogeneous agents, representing classes of agents with low, medium or high incomes.

Each agent is endowed with an initial portfolio $\left(0, \theta_{-}^{h}\right)$ of the riskless bond and the available stocks, ${ }^{5}$ with current income, representing current labor income plus dividends from $\theta_{-}^{h}, e_{0}^{1}=2 / 3, e_{0}^{2}=1$, and $e_{0}^{3}=4 / 3$, and with stochastic future labor income given by some $l^{h} \in \mathbb{R}_{++}^{S}$. We are back in the framework of Section 2 by setting $e_{0}^{1}=2 / 3, e_{0}^{2}=1$ and $e_{0}^{3}=4 / 3$, and $\widetilde{e}^{h}=l^{h}+\sum_{j=2}^{J} \theta_{-j}^{h} d^{j}$ for $h=1, \ldots, H$. For each household $h$, the labor incomes $l_{s}^{h}$ are generated by $S$ independent draws from some given distribution. In this way we can obtain a discrete approximation of any continuous distribution.

The first agent has no capital income, $\theta_{-}^{1}=0$. For the other agents we have $\theta_{-}^{2}=$ $1 / 3 \cdot 1_{J-1}$ and $\theta_{-}^{3}=2 / 3 \cdot 1_{J-1}$. In most applications agents have heterogeneous von Neumann-Morgenstern utility functions with identical uniform probabilities over states and identical discount factors $\delta^{h}=0.95$.

The assets available are given by a riskless bond and seven stocks. In most examples the dividends of asset $j$ depend on a single common factor $f \in \mathbb{R}^{S}$ as well as on an idiosyncratic shock $\varepsilon^{j} \in \mathbb{R}^{S}$. We denote asset $j$ 's load in the factor by $c_{j}$, varying from 0.25 to 1.75 in steps of 0.25 . The examples are calibrated to yearly US data. The expected growth rate of aggregate consumption equals two percent and the standard deviation of both the factor and the idiosyncratic shock determining the dividends are about 0.13 - giving an overall standard deviation of the stock market of about 0.17 . The standard deviation of labor income is chosen to be around 0.10 and labor income constitutes around $2 / 3$ of total income. The eleven random variables in the model are therefore $\left(\left(l^{h}\right)_{h=1, \ldots, H}, f,\left(\varepsilon^{j}\right)_{j=2, \ldots, J}\right)$.

As a first example we analyze the case where the realization of each random variable is either high or low with equal probabilities, and all random variables are independent. The minimal state space to achieve this consists of $2^{11}=2,048$ states. More specifically we have that

$$
\begin{aligned}
l_{s}^{h} & \in\{2 / 3 \cdot(1.02-0.1), 2 / 3 \cdot(1.02+0.1)\}, \\
f_{s} & \in\{-0.13,0.13\}, \\
\varepsilon_{s}^{j} & \in\{-0.13,0.13\} .
\end{aligned}
$$

Dividends of asset $j$ are then determined by

$$
d_{s}^{j}=1 / 3 \cdot 1 / 7 \cdot\left(1.02+\sqrt{c_{j}} f_{s}+\varepsilon_{s}^{j}\right) .
$$

We assume that all agents have constant relative risk aversion utility functions of the form

$$
v^{h}\left(c_{s}^{h}\right)=\frac{\left(c_{s}^{h}\right)^{1-\gamma^{h}}}{1-\gamma^{h}}, \quad c_{s}^{h}>0,
$$

where $\gamma^{h}$ is the coefficient of relative risk aversion. We choose $\gamma^{1}=6, \gamma^{2}=4$ and $\gamma^{3}=2$.

With these specifications we compute the equilibrium prices and portfolio-holdings and compare them to the predictions of the CAPM in Figure 1. To do those computations, we

\footnotetext{
${ }^{5}$ Note that contrary to the model described in Section 2, we assume now that stocks are in unit net supply.
} 
could in principle use the homotopy algorithms as reported in Brown, DeMarzo and Eaves (1996) or Schmedders (1998), which can solve for an equilibrium in the general multiple commodities GEI-model. The problem is that for both algorithms the number of equations to be solved is a multiple of the number of states, whereas the number of states is 1,024 for the current economy and 32,768 for the other economies considered in this paper. This makes both algorithms unsuitable for our purposes. In Herings and Kubler (2000) we develop an algorithm that is tailored to the finance GEI-model with one good per state, and that is independent of the number of states. Instead, the number of equations to be solved is related to the number of assets, which is 8 for most economies analyzed in this paper. Our algorithm therefore allows for an effective and efficient computation in finance economies, which is imperative to address the issues in this paper.

The solid line in the figure is the security market line, i.e. the CAPM relationship between a portfolio's $\beta$ and its risk premium. The actual equilibrium expected returns of the seven securities are depicted by + and lie all almost exactly on the security market line. CAPM turns out to be an extraordinarily good predictor for the actual equilibrium returns of assets in this example. This is surprising as preferences are far from mean-variance, and asset returns are far from being normally distributed.

Although the graph of Figure 1 looks very convincing, it is clear that we need more objective measures to quantify the deviation of equilibrium prices and portfolio-holdings from the CAPM predictions. Note that we need to check both the robustness of two-fund separation and the robustness of the pricing-formula. With general preferences CAPMpricing is neither necessary nor sufficient for two-fund separation. It is easy to see that twofund separation does not imply CAPM-pricing. Consider a model with complete markets where all agents have identical constant absolute risk aversion preferences. It is well known that two-fund separation holds since there exists a linear sharing rule, see also Cass and Stiglitz (1970). However, it is easy to see that generally

$$
\pi_{A}^{*}=\operatorname{proj}_{\langle A\rangle}\left(\frac{\delta^{h} \partial_{c_{1}^{h}} v_{1}^{h}\left(e_{1}\right)}{\partial_{c_{0}^{h}} v_{0}^{h}\left(e_{0}\right)}, \ldots, \frac{\delta^{h} \partial_{c_{S}^{h}} v_{S}^{h}\left(e_{S}\right)}{\partial_{c_{0}^{h}} v_{0}^{h}\left(e_{0}\right)}\right)=\left(\frac{\delta^{h} \partial_{c_{1}^{h}} v_{1}^{h}\left(e_{1}\right)}{\partial_{c_{0}^{h}} v_{0}^{h}\left(e_{0}\right)}, \ldots, \frac{\delta^{h} \partial_{c_{S}^{h}} v_{S}^{h}\left(e_{S}\right)}{\partial_{c_{0}^{h}} v_{0}^{h}\left(e_{0}\right)}\right) \notin\left\langle 1_{S}, \widetilde{e}\right\rangle .
$$

Conversely, suppose prices of assets consistent with CAPM-pricing are given, and let asset markets be complete for simplicity. It is easy to choose individual consumption bundles which do not belong to $\left\langle 1_{S}, \widetilde{e}\right\rangle$ and utility functions for which the individual consumption bundles are optimal at the asset prices chosen.

The most straightforward approach is to measure the accuracy of CAPM-pricing is to take the Mean Squared Error (MSE), which is defined by

$$
\mathrm{MSE}=\sqrt{\frac{1}{J-1} \sum_{j=2}^{J}\left(r_{j}^{*}-\widehat{r}_{j}\right)^{2}}
$$

where $r_{j}^{*}$ denotes the equilibrium expected return of asset $j$ and $\widehat{r}_{j}$ the prediction by CAPM.

A different approach consists of the following. By the arguments used in the proof of Theorem 2.3 it is obvious that $\pi_{A}^{*} \in\left\langle 1_{S}, \widetilde{e}_{\mathrm{M}}\right\rangle$ is sufficient for CAPM-pricing. That 


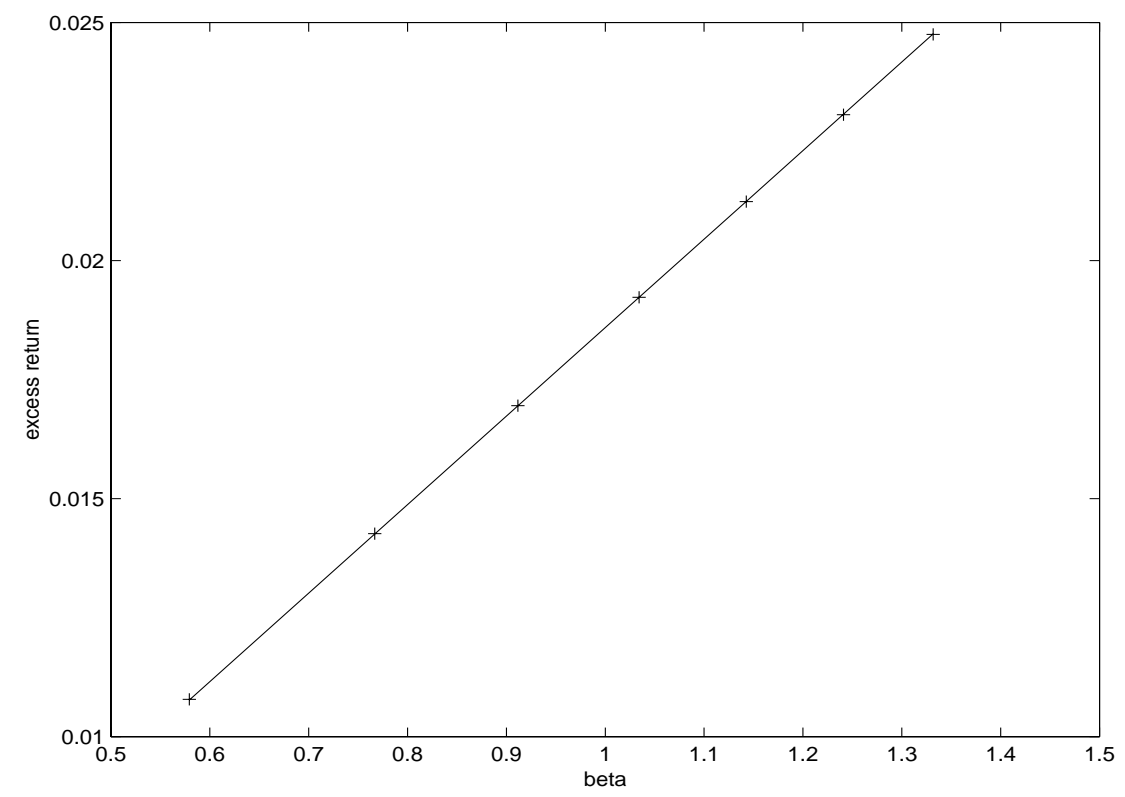

FiguRE 1: Security market line with high-low returns. 
this is necessary as well follows from the observation that otherwise $\pi_{A}^{*}$ is equal to the sum of its projection on $\left\langle 1_{S}, \widetilde{e}_{\mathrm{M}}\right\rangle$ plus a non-zero orthogonal part in $\langle A\rangle$ under the inner product $\rho$. When CAPM-pricing is valid, the orthogonal part should have zero price, which is obviously not the case when priced by $\pi_{A}^{*}$. Therefore, an interesting alternative to MSE is to take the OLS $R^{2}$ of the regression with

$$
\operatorname{proj}_{\langle A\rangle}\left(\frac{\partial_{c_{1}^{h}} u^{h}\left(c^{h *}\right) / \rho_{1}}{\partial_{c_{0}^{h}} u^{h}\left(c^{h *}\right)}, \ldots, \frac{\partial_{c_{S}^{h}} u^{h}\left(c^{h *}\right) / \rho_{s}}{\partial_{c_{0}^{h}} u^{h}\left(c^{h *}\right)}\right)
$$

as regressand and $1_{S}$ and $\widetilde{e}_{\mathrm{M}}$ as regressors. Notice that this measure is independent of $h$. We call it Pricing $R^{2}$.

To measure how well two-fund separation holds for agent $h$, we take the OLS $R^{2}$ of the regression with $\left(\theta_{j}^{h *}\right)_{j=2, \ldots, J}$ as regressand and $\widehat{\theta}_{\mathrm{M}}$, the risky part of the market portfolio, as regressor.

The following table confirms that CAPM provides an outstanding prediction for the economy under consideration.

\begin{tabular}{|l|l|}
\hline$R^{\mathrm{f}}$ & 1.0633 \\
\hline Equity Premium & 0.0185 \\
\hline MSE & 0.0000530 \\
\hline Pricing $R^{2}$ & 0.99999998 \\
\hline Two-fund $R^{2} h=1$ & 0.9999988 \\
Two-fund $R^{2} h=2$ & 0.9999994 \\
Two-fund $R^{2} h=3$ & 0.9999998 \\
\hline
\end{tabular}

TABLE 1: CAPM for CRRA preferences and two-point distributions.

Although the high-low specifications for the random variables are two-point approximations to normal random variables the well-known fact that CAPM holds with normally distributed returns does not imply anything about the validity of CAPM in this framework. It is easy to see that two-point approximations to normal random variables do not satisfy the properties of elliptical distributions. The following trivial example shows that while each dividends distribution is characterized by its mean and variance it is not true that a linear combination of these random variables is also fully characterized by its mean and variance.

EXAMPle 3.2: Consider a model with 4 states where all probabilities are equal. Let

$$
d^{1}=\left(\begin{array}{c}
-0.5 \\
-0.5 \\
0.5 \\
0.5
\end{array}\right), \quad d^{2}=\left(\begin{array}{c}
0.5 \\
-0.5 \\
0.5 \\
-0.5
\end{array}\right)
$$


Both $d^{1}$ and $d^{2}$ are discrete distributions such that with probability $1 / 2$ the realization is -0.5 and with probability $1 / 2$ the realization is 0.5 . However, both $d^{1}+d^{2}$ and $\sqrt{2} d^{1}$ have expectation zero and variance $1 / 2$, but correspond to different distributions and different utilities when the utility function is not mean-variance.

One should not expect CAPM to hold in this model even though the distributions provide a (very crude) approximation to normal distributions.

Also the fact that two-fund separation holds so well, comes as a surprise. Since the households we are dealing with have different parameters of relative risk aversion, there is no reason to expect that two fund separation obtains, see Cass and Stiglitz (1970) and Detemple and Gottardi (1998).

\section{Robustness in Preferences}

In order to show that the predictions of CAPM are a good approximation for equilibria in a wide variety of economic settings we compute 600 examples. We assume that there are $S=32,768$ states of nature. Using a large number of states guarantees that our final samples are good approximations of continuous distributions. By taking a large number of states we rule out finite sample effects on the prices of assets. When we replicate the experiment and generate economies out of a newly drawn sample, the equilibrium will be almost the same if the number of states is sufficiently large.

Throughout this section we assume that all random variables are log-normally distributed, so $l_{s}^{h}, f_{s}$, and $\varepsilon_{s}^{j}$ are drawn independently from a log-normal distribution. The log-normal distribution with mean $\mu$ and variance $\sigma^{2}$ is denoted by $\operatorname{LN}\left(\mu, \sigma^{2}\right)$. Since we are considering finite samples, the drawing will be of (some) influence on the equilibrium we compute. As before asset 1 is the riskless bond. For $j \geq 2$, we define asset $j$ 's dividend to be

$$
d_{s}^{j}=1 / 3 \cdot 1 / 7 \cdot 1.02 \cdot f_{s}^{j} \cdot \varepsilon_{s}^{j}
$$

and we choose

$$
\begin{aligned}
l_{s}^{h} & \sim \operatorname{LN}\left(2 / 3 \cdot 1.02,(2 / 3)^{2} \cdot 0.01\right) \\
f_{s}^{j} & \sim \operatorname{LN}\left(1, c_{j} \cdot 0.0161\right) \\
\varepsilon_{s}^{j} & \sim \operatorname{LN}(1,0.0161)
\end{aligned}
$$

The actual $\left(f_{s}^{j}\right)_{j=2}^{J}$ are all based on a single realization of a normal random variable $\widehat{f}_{s}$. For each asset $j$, we linearly transform the realization of this random variable in such a way that after taking the exponent a log-normally distributed random variable with mean 1 and variance $c_{j} \cdot 0.0161$ results. The construction of the random variables implies that all dividends themselves are log-normally distributed. To get a similar variance of the entire stock market as before the variance of the factors and the idiosyncratic shock have 
to be chosen to be 0.0161 instead of 0.0169 . Notice that the factor realization does not enter linearly in the formula for the asset's dividends, an assumption that is made in most models describing factor economies. This is an additional advantage as it puts CAPM only more seriously to the robustness test. Finally, it follows from the work of Feldstein (1969) that log-normal distributions do not belong to the elliptic class, and would not admit of two-fund separation.

We consider three different families of utility functions and compute fifty randomly generated examples within each class. For each class we report histograms of the MSE, the Pricing $R^{2}$, and the Two-fund $R^{2}$ of agent 2 and 3. By market clearing, the portfolioholdings of agent 1 are fully dependent on those of agents 2 and 3. If two-fund separation holds exactly for agents 2 and 3 it will hold exactly for agent 1 as well. Therefore, we safe space and do not report the Two-fund $R^{2}$ of agent 1 . In all histograms the scaling is taken identically, so that results for different models can be compared easily.

We first assume that all agents' utility functions exhibit constant absolute risk aver, i.e.

$$
v^{h}\left(c_{s}^{h}\right)=-\exp \left(\alpha^{h} c_{s}^{h}\right), \quad c_{s}^{h} \in \mathbb{R}
$$

where $\alpha^{h}$ is the coefficient of absolute risk aversion. We then move on and examine an economy where all agents' utility functions exhibit constant relative risk aversion, i.e.

$$
\begin{aligned}
& v^{h}\left(c_{s}^{h}\right)=\frac{\left(c_{s}^{h}\right)^{1-\gamma^{h}}}{1-\gamma^{h}}, \quad c_{s}^{h}>0, \quad \gamma^{h} \neq 1, \\
& v^{h}\left(c_{s}^{h}\right)=\log \left(c_{s}^{h}\right), \quad c_{s}^{h}>0, \quad \gamma^{h}=1,
\end{aligned}
$$

where $\gamma^{h}$ is the coefficient of relative risk aversion.

The rationale for examining both constant absolute risk aversion and constant relative risk aversion is as follows. Kenneth Arrow has repeatedly argued that it is realistic assume increasing absolute risk aversion and non-increasing relative risk aversion. By covering the two extreme cases of constant absolute and constant relative risk aversion we want to argue that CAPM provides a good approximation for pricing for all specifications which satisfy Arrow's criteria.

\subsection{Random CARA}

We randomly generate 100 examples of economies where all agents have constant absolute risk aversion. For each example we draw the coefficient of risk aversion $\alpha^{h}, h=1,2,3$, from a uniform distribution on the interval $[0.5,10]$. Comparisons between the computed equilibria and the CAPM predictions are depicted in the histograms of Figures $2 \mathrm{a}-\mathrm{d} .{ }^{6}$

Obviously CAPM predicts extremely well. The mean squared error always lies below 0.04 percent. In most cases it is around $0.5 \cdot 10^{-4}$. The Pricing $R^{2}$ exceeds 0.9999 in all

\footnotetext{
${ }^{6}$ The Pricing $R^{2}$ is multiplied by 100 to avoid round-off to 1.000 by our software.
} 


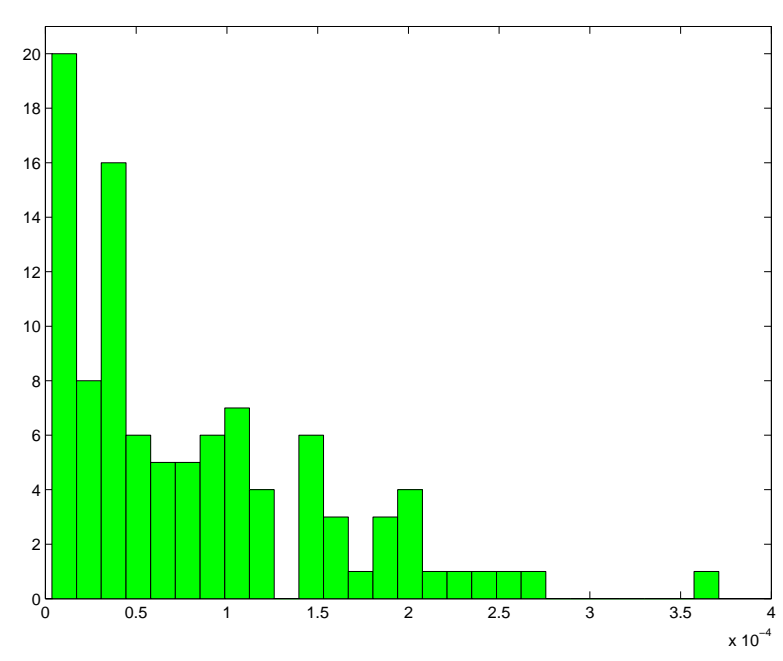

Figure 2A: CARA: MSE.

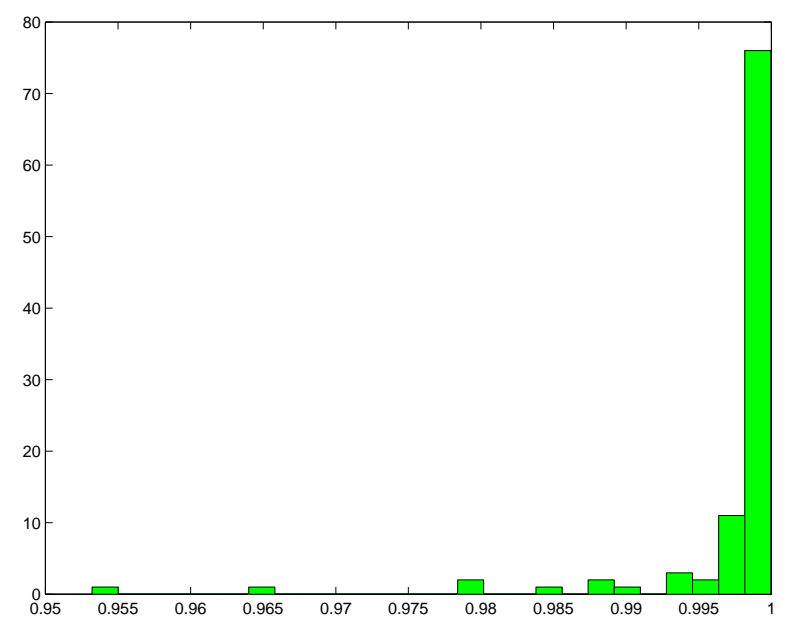

Figure 2C: CARA: Two-fund separation agent 2 .

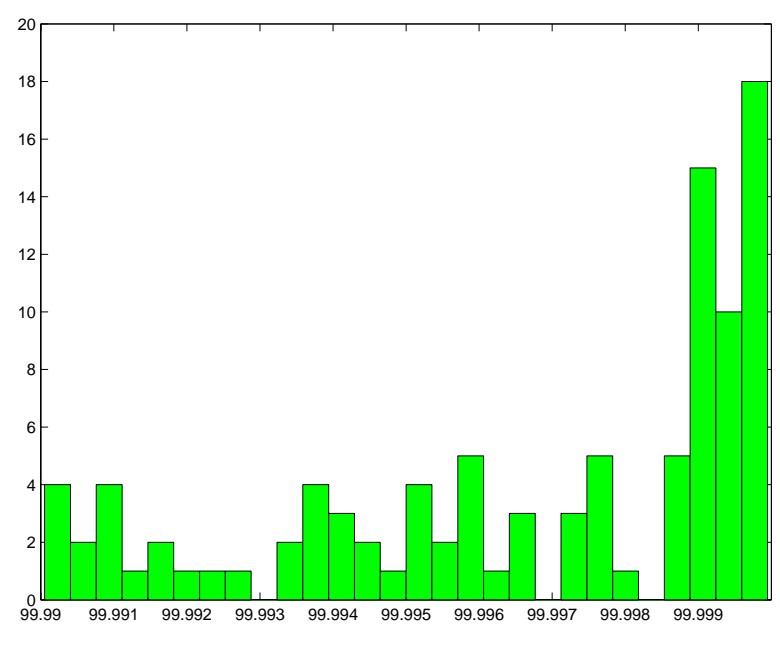

Figure 2B: CARA: 100 . Pricing $R^{2}$.

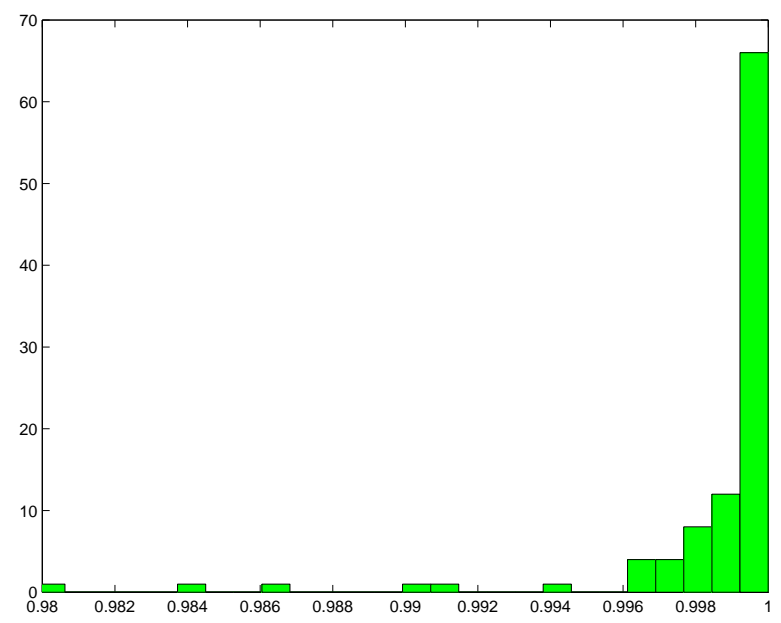

Figure 2D: CARA: Two-fund separation agent 3 . 
examples. The Two-fund $R^{2}$ exceeds 0.99 in most cases. Compared to the single example examined in Section 3, the results are slightly worse on average. Figure 3 clarifies that this can be entirely explained by higher values for the average rate of risk aversion present in the economy. The MSE increases with average risk aversion in the economy, as measured by the harmonic mean of the $\alpha^{h}$ 's (it is well known that the harmonic mean is the right measure for average risk aversion in an economy where all agents have constant absolute risk aversion).

Although CAPM remains an excellent predictor for all cases examined so far, Figure 3 indicates that CAPM is a better tool in environments with lower average risk aversion. In the light of this result one might be tempted to draw a parallel between our results and the observation of Mehra and Prescott (1985) that realistic values of risk-aversion do not produce a realistic equilibrium risk-premium. If the equilibrium returns of risky assets do not change significantly with small variations of agents' coefficient of relative risk aversion it can be expected that the cross section remains almost unchanged and that CAPM (which predicts excess returns independently of preferences) provides a good prediction for a variety of attitudes towards risk. Note, however, that this can only explain one side of the phenomena - the question remains why the cross-section of returns can be described by the assets' $\beta$ 's.

\subsection{RANDOM CRRA}

We now assume that all agents have constant relative risk aversion and we draw $\gamma^{h}, h=$ $1,2,3$, from a uniform distribution on the interval $[0.5,10]$. With mean household income equal to 1 , the degree of risk-aversion in the economy is similar to the CRRA-case examined in Subsection 4.1. As before we compute 100 examples - Figures 4a-d report the analogues of Figures 2a-d for the CRRA case.

Figure 4 shows that CAPM is an excellent predictor for the class of CRRA utility functions, both in terms of pricing and in terms of two-fund separation. In most cases MSE is around $1 \cdot 10^{-4}$. The worst Pricing $R^{2}$ found is 0.99995 and the worst Two-fund $R^{2}$ is 0.95 .

The high values of the Pricing $R^{2}$ provides very useful information for the pricing of assets. Recall that the price of asset $j$ is given by $\pi_{A}^{*} \cdot d^{j}$. Any vector that is highly correlated with $\pi_{A}^{*}$ should lead to a similar price for asset $j$. In particular, when the Pricing $R^{2}$ is close to one, CAPM is bound to give almost exact equilibrium prices and the use of CAPM leads to a low MSE.

\subsection{Loss Aversion}

To demonstrate that our results do not depend on state independent utility, we analyze a class of utility functions that are state dependent and that are characterized by loss

aversion. Such utility functions get support from empirical work on the decision making 


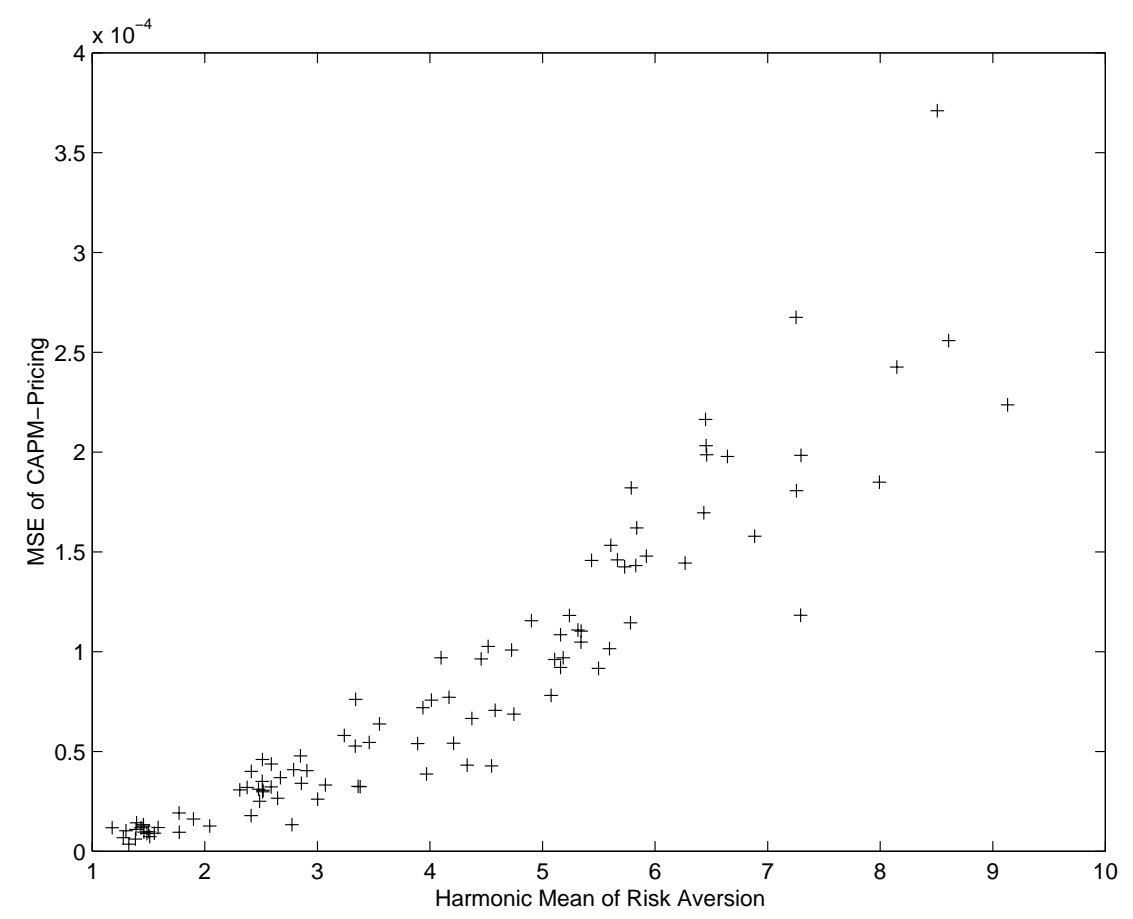

FiguRE 3: MSE against Risk-Aversion for CRRA preferences. 


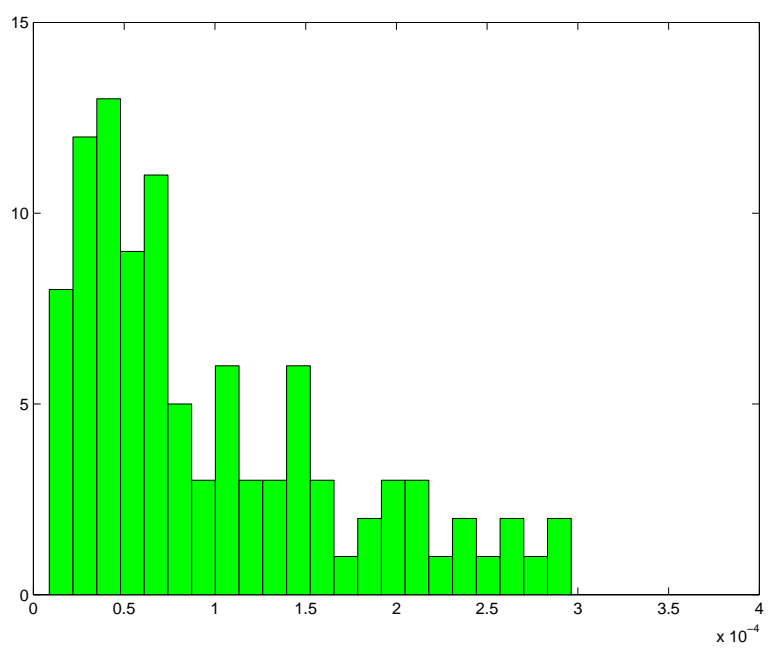

Figure 4A: CRRA: MSE.

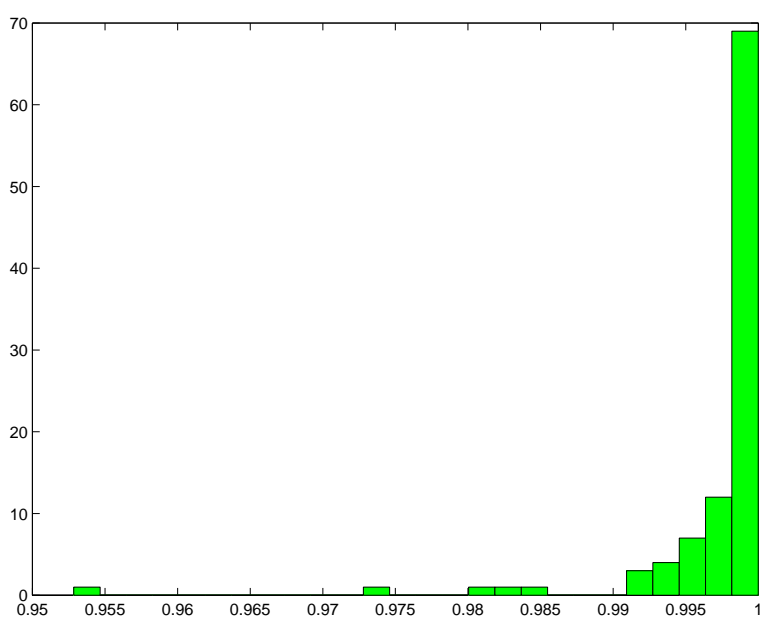

Figure 4C: CRRA: Two-fund separation agent 2.

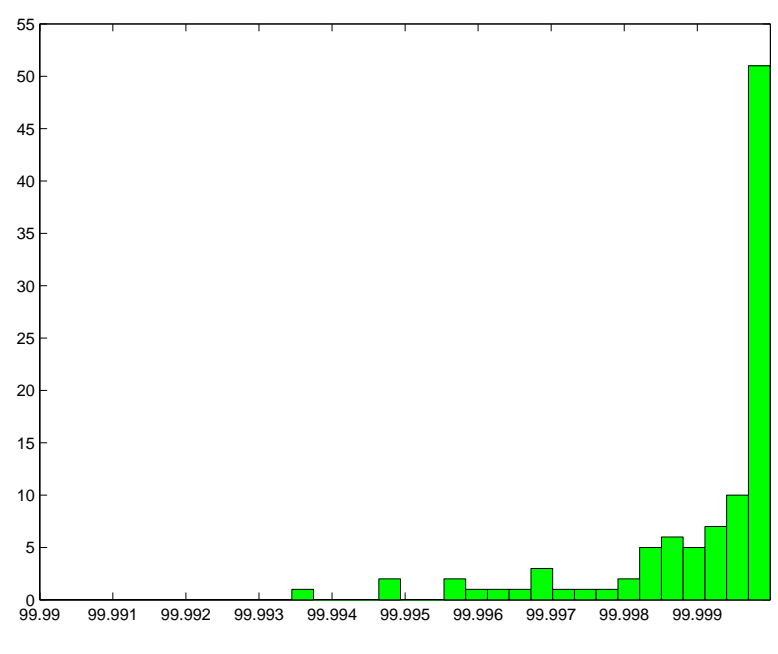

Figure 4B: CRRA: 100. Pricing $R^{2}$.

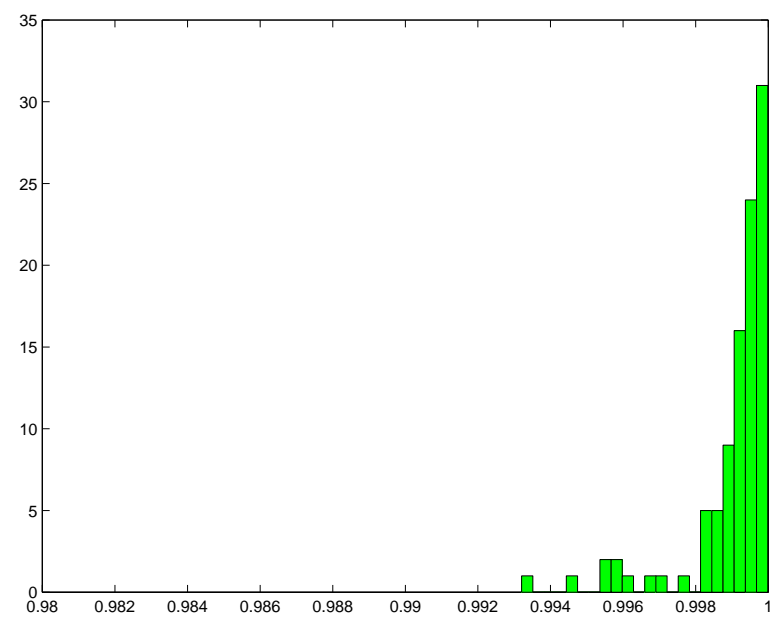

Figure 4D: CRRA: Two-fund separation agent 3 . 
of agents. They are also claimed to be helpful in explaining the equity premium puzzle of Mehra and Prescott (1985), see Benartzi and Thaler (1995).

We cannot use exactly the same utility functions as Benartzi and Thaler, as these are not everywhere quasi-concave, and as a consequence a competitive equilibrium may not exist. The important characteristic of loss-aversion is not so much the existence of nonconcavities, but a sharp decrease in utility when loosing income compared to the status quo and only a mild increase in utility when gaining income. This is usually modeled by a utility function that has a kink at the status quo.

We generate a utility function with loss aversion as follows. We identify the status quo of an agent $h$ in state $s \geq 1$ with $e_{s}^{h}$. Then loss aversion applies to making good or bad investment decisions on the stock market. Consistent with Benartzi and Thaler (1995), we want a Bernoulli function $v_{s}^{h}$ such that $\lim _{c_{s}^{h} \uparrow e_{s}^{h}} \partial v_{s}^{h}\left(c_{s}^{h}\right)=2 \lim _{c_{s}^{h} \downarrow e_{s}^{h}} \partial v_{s}^{h}\left(c_{s}^{h}\right)$. For each $h$, we choose parameters $\gamma_{1}^{h}$ and $\gamma_{2}^{h}$. When $c_{s}^{h} \geq e_{s}^{h}$, then $v_{s}^{h}$ coincides with a CRRA utility function with parameter of relative risk aversion $\gamma_{1}^{h}$. When $c_{s}^{h} \leq e_{s}^{h}$, then $v_{s}^{h}$ coincides with a CRRA utility function with parameter of relative risk aversion $\gamma_{2}^{h}$, plus a term linear in $c_{s}^{h}$ to get $\lim _{c_{s}^{h} \uparrow e_{s}^{h}} \partial v_{s}^{h}\left(c_{s}^{h}\right)=2 \lim _{c_{s}^{h} \downarrow e_{s}^{h}} \partial v_{s}^{h}\left(c_{s}^{h}\right)$, plus a constant $k_{s}$ to make $v_{s}^{h}$ continuous. More precisely, we assume that $u^{h}\left(c^{h}\right)=v_{0}^{h}\left(c_{0}^{h}\right)+\delta^{h} \sum_{s=1}^{S} \rho_{s} v_{s}^{h}\left(c_{s}^{h}\right)$, where

$$
\begin{aligned}
& v_{0}^{h}\left(c_{0}^{h}\right)=\left(c_{0}^{h}\right)^{1-\gamma_{1}^{h}} /\left(1-\gamma_{1}^{h}\right), \\
& v_{s}^{h}\left(c_{s}^{h}\right)=\left(c_{s}^{h}\right)^{1-\gamma_{2}^{h}} /\left(1-\gamma_{2}^{h}\right)+\left(\frac{\gamma_{2}}{\gamma_{1}}\left(e_{s}^{h}\right)^{-\gamma_{1}^{h}}-\left(e_{s}^{h}\right)^{-\gamma_{2}^{h}}\right) c_{s}^{h}+k_{s}, \quad c_{s}^{h} \leq e_{s}^{h}, \\
& v_{s}^{h}\left(c_{s}^{h}\right)=\left(c_{s}^{h}\right)^{1-\gamma_{1}^{h}} /\left(1-\gamma_{1}^{h}\right), \quad c_{s}^{h} \geq e_{s}^{h},
\end{aligned}
$$

with

$$
k_{s}=\frac{\gamma_{2}}{1-\gamma_{2}}\left(e_{s}^{h}\right)^{1-\gamma_{2}^{h}}+\frac{\gamma_{1}^{h} \gamma_{2}^{h}+\gamma_{1}^{h}-\gamma_{2}^{h}}{\gamma_{1}^{h}\left(1-\gamma_{1}^{h}\right)}\left(e_{s}^{h}\right)^{1-\gamma_{1}^{h}} .
$$

The Bernoulli function $v_{s}^{h}$ is continuous and is continuously differentiable except at $e_{s}^{h}$ where it has a kink. It can be shown that the coefficient of relative risk aversion varies continuously in $c_{s}^{h}$ and is given by $\left(\gamma_{1}^{h} \gamma_{2}^{h}\left(c_{s}^{h}\right)^{-\gamma_{2}^{h}}\right) /\left(\gamma_{2}^{h}\left(e_{s}^{h}\right)^{-\gamma_{1}^{h}}-\gamma_{1}^{h}\left(e_{s}^{h}\right)^{-\gamma_{2}^{h}}+\gamma_{1}^{h}\left(c_{s}^{h}\right)^{-\gamma_{2}^{h}}\right)$ if $c_{s}^{h} \leq e_{s}^{h}$, so it approaches $\gamma_{2}^{h}$ as $c_{s}^{h} \rightarrow 0$. The coefficient of relative risk aversion is given by $\gamma_{1}^{h}$ if $c_{s}^{h} \geq e_{s}^{h}$.

Since $v_{s}^{h}$ is not differentiable at $e_{s}^{h}$ it does not satisfy the assumptions under which the algorithm has been shown to be convergent. We have to smooth out the kinks of the utility function. We can do this by taking any $e_{s}^{h-}, e_{s}^{h+}$ such that $e_{s}^{h-}<e_{s}^{h}<e_{s}^{h+}$ and defining

$$
\partial v_{s}^{h}\left(c_{s}^{h}\right)=\frac{e_{s}^{h+}-c_{s}^{h}}{e_{s}^{h+}-e_{s}^{h-}} \partial v_{s}^{h}\left(e_{s}^{h-}\right)+\frac{c_{s}^{h}-e_{s}^{h-}}{e_{s}^{h+}-e_{s}^{h-}} \partial v_{s}^{h}\left(e_{s}^{h+}\right) .
$$

In principle, the parameter $k_{s}$ has to be adjusted to make $v_{s}^{h}$ continuous. Since our algorithm works entirely with first order conditions, this is of no concern to us. In the numerical experiments we took $e_{s}^{h-}=0.95 e_{s}^{h}$ and $e_{s}^{h+}=1.05 e_{s}^{h}$. For each example we take $\gamma_{1}^{h}=\gamma_{2}^{h} / 2$ and we draw $\gamma_{2}^{h}, h=1,2,3$, from a uniform distribution on the interval $[1,6]$. In this way 


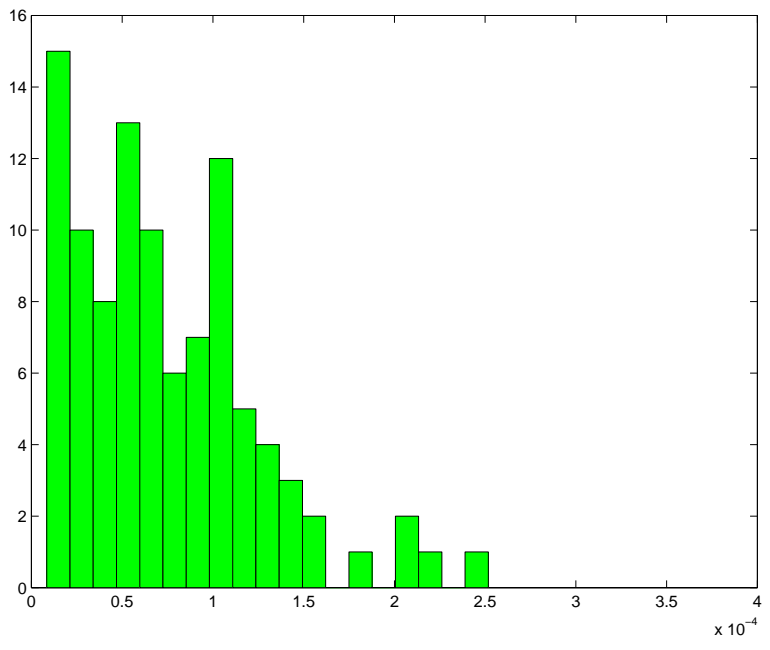

Figure 5A: LA: MSE.

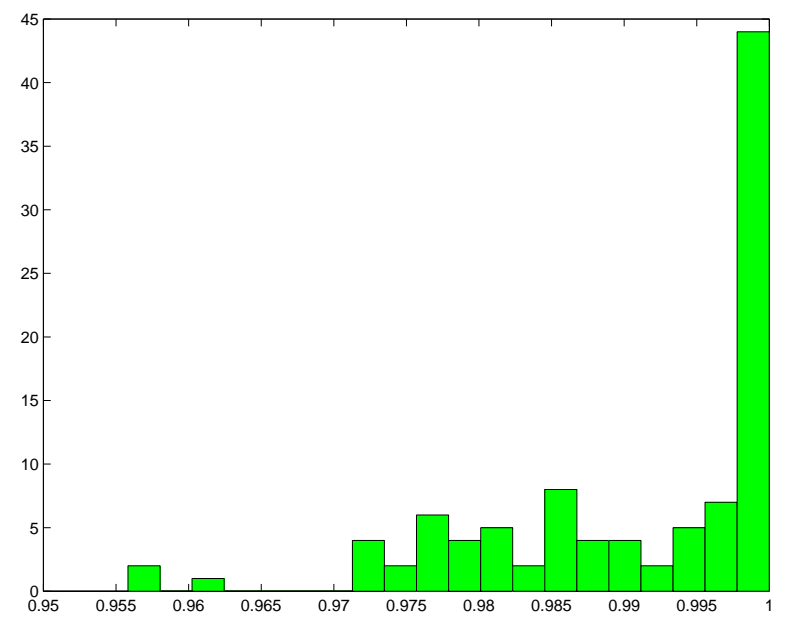

Figure 5C: LA: Two-fund separation agent 2.

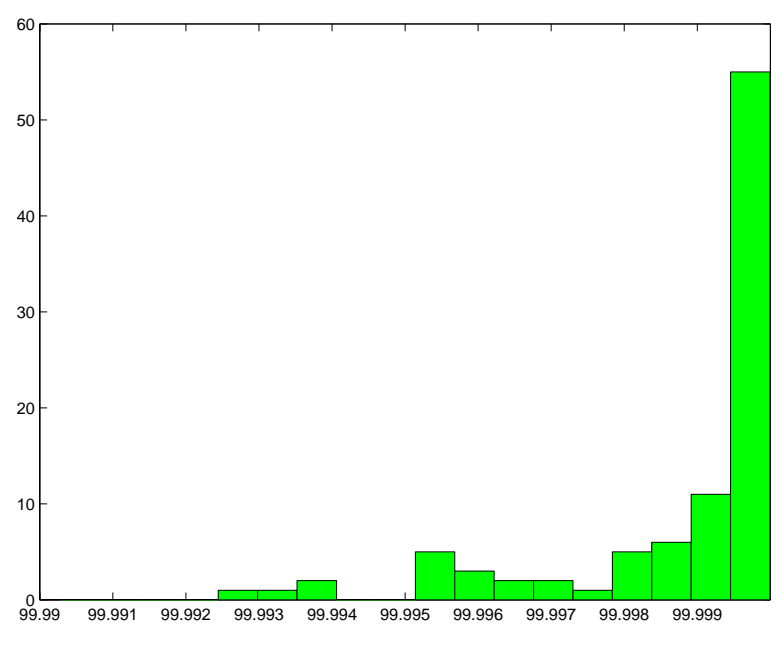

Figure 5B: LA: 100. Pricing $R^{2}$.

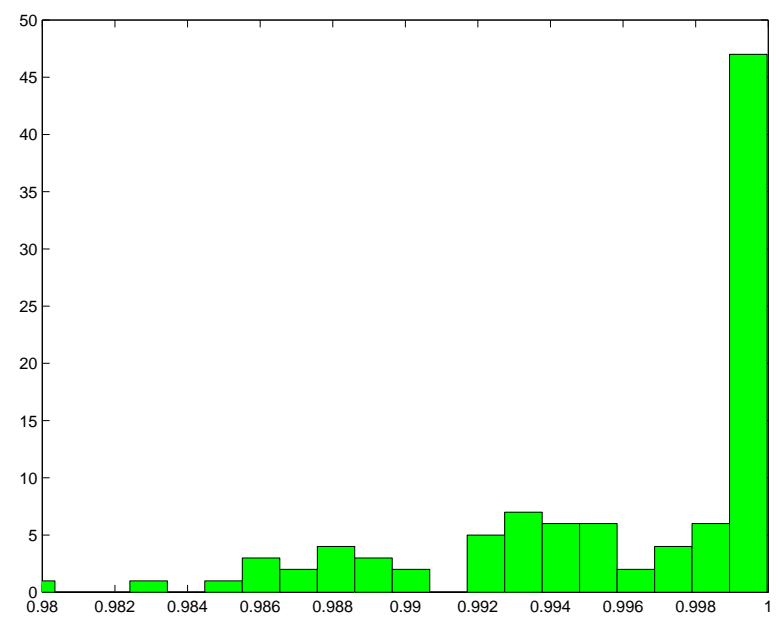

Figure 5D: LA: Two-fund separation agent 3 . 
fifty economies are randomly generated. The outcomes of our computations are presented in Figures 5a-d.

It turns out that CAPM is an extraordinarily good predictor for the case with loss aversion. The results seem to be even better than for the CRRA and CARA cases examined before. In most cases, MSE is below $1 \cdot 10^{-4}$, Pricing $R^{2}$ exceeds 0.99999 , and the Two-fund $R^{2}$ exceeds 0.98 . If we take into account that the examples with loss aversion are such that the degree of risk aversion is lower on average than before, the Pricing $R^{2}$ is comparable to the one found for CRRA and CARA preferences.

\section{Robustness in Return Processes}

We now fix agents' preferences to exhibit constant relative risk aversion and choose $\gamma^{1}=6$, $\gamma^{2}=4$, and $\gamma^{3}=2$. We test the robustness of our results to variations in the distributions of endowments and assets. We consider three different families of return processes and compute 100 randomly generated examples within each class. We show the histograms of MSE, Pricing $R^{2}$, and Two-fund $R^{2}$ of agents 2 and 3 .

\subsection{Uniform RETURNS}

In order to verify whether our results depend on the assumption of log-normal shocks, we now assume that all shocks are uniformly distributed. We also allow for some variation in the ratio of labor income to total income, in the variance of the factor and in the variance of the idiosyncratic shocks.

We start each example by randomly generating parameters $a_{1}, a_{2}, a_{3}$ and $a_{4}$, where

$$
\begin{aligned}
& a_{1} \sim \mathrm{U}(1.02 \cdot 0.5,1.02 \cdot 0.9), \\
& a_{2} \sim \mathrm{U}(1.02 \cdot 1.1,1.02 \cdot 1.5), \\
& a_{3} \sim \mathrm{U}(-0.5,-0.1), \\
& a_{4} \sim \mathrm{U}(0.1,0.5) .
\end{aligned}
$$

Given a realization for $a_{1}, \ldots, a_{4}$, we continue the construction of the economy by taking independent drawings for $l_{s}^{h}, f_{s}$ and $\varepsilon_{s}^{j}$, where

$$
\begin{aligned}
l_{s}^{h} & \sim \mathrm{U}(2 / 3 \cdot 0.8,2 / 3 \cdot 1.24), \\
f_{s} & \sim \mathrm{U}\left(\left(a_{1}-a_{2}\right) / 2,\left(a_{2}-a_{1}\right) / 2\right), \\
\varepsilon_{s}^{j} & \sim \mathrm{U}\left(a_{3}, a_{4}\right) .
\end{aligned}
$$

Finally, dividends are determined by

$$
d_{s}^{j}=1 / 3 \cdot 1 / 7 \cdot\left(\frac{a_{1}+a_{2}}{2}+\sqrt{c_{j}} f_{s}+\varepsilon_{s}^{j}\right) .
$$

Given the realizations for the parameters $a_{1}$ and $a_{2}, 1 / 3 \cdot 1 / 7 \cdot\left(a_{1}+a_{2}\right) / 2$ equals expected dividends from asset $j$. The realization of the factor belongs to the interval $\left[\left(a_{1}-a_{2}\right) / 2,\left(a_{2}-\right.\right.$ 
$\left.a_{1}\right) / 2$ ] and the realizations of the idiosyncratic shocks to the interval $\left[a_{3}, a_{4}\right]$. The expected labor income and the variance of labor income are taken as before.

Figures $6 \mathrm{a}-\mathrm{d}$ show that the ability of CAPM to predict portfolio-holdings and excess returns is robust to the exact specification of the distribution of shocks. The results are very close to the ones obtained for the base case with log-normal shocks examined in Section 3, where the average degree of risk aversion in the economy is similar.

\subsection{MORE FACTORS}

One might wonder whether our results are not simply due to the fact that we have all risky assets being influenced by a single common factor. In fact, it is possible to derive CAPM as a special case of APT where there is only one factor, see for instance Connor (1984). However, such a derivation requires an uncountable number (or at least very large number) of assets to diversify the idiosyncratic shocks away. The importance of idiosyncratic shock is quite substantial in our economies with only seven risky assets. Moreover, usually factors enter linearly in the definition of an asset's pay-off, which is not always the case in our economies. It seems therefore not likely that our results are due to the single factor set-up.

Other suspicious elements of the set-up we used so far are that factor loads are distributed very symmetrically and balanced, and that the importance of idiosyncratic shocks is the same for all assets. Finally, we consider a wider range for the variance of the entire stock market.

In this subsection we generate a number of economies where risky assets depend on two factors, $f$ and $\widehat{f}$, and factor loads for each one of the assets are randomly drawn. On top of this, also the importance of the idiosyncratic shock is randomly determined.

We start each example by randomly generating, for each asset $j=2, \ldots, J$, parameters $c_{j}, \widehat{c}_{j}$, and $i_{j}$. These parameters represent the load in factor 1 , the load in factor 2 and the importance of the idiosyncratic shock. More specifically it holds that

$$
\begin{aligned}
c_{j} & \sim \mathrm{U}(0,2), \\
\widehat{c}_{j} & \sim \mathrm{U}(0,2), \\
i_{j} & \sim \mathrm{U}(0,4) .
\end{aligned}
$$

Labor income, the two factors and assets' idiosyncratic shocks are independently lognormally distributed, so $l_{s}^{h}, f_{s}, \widehat{f}_{s}$, and $\varepsilon_{s}^{j}$ are drawn from a log-normal distribution,

$$
\begin{aligned}
l_{s}^{h} & \sim \operatorname{LN}\left(2 / 3 \cdot 1.02,(2 / 3)^{2} \cdot 0.01\right) \\
f_{s}^{j} & \sim \operatorname{LN}\left(1, c_{j} \cdot 0.0161\right) \\
\widehat{f}_{s}^{j} & \sim \operatorname{LN}\left(1, \widehat{c}_{j} \cdot 0.0161\right) \\
\varepsilon_{s}^{j} & \sim \operatorname{LN}\left(1, i_{j} \cdot 0.0161\right) .
\end{aligned}
$$

Finally, dividends are determined by

$$
d_{s}^{j}=1 / 3 \cdot 1 / 7 \cdot 1.02 \cdot f_{s}^{j} \cdot \widehat{f}_{s}^{j} \cdot \varepsilon_{s}^{j} .
$$




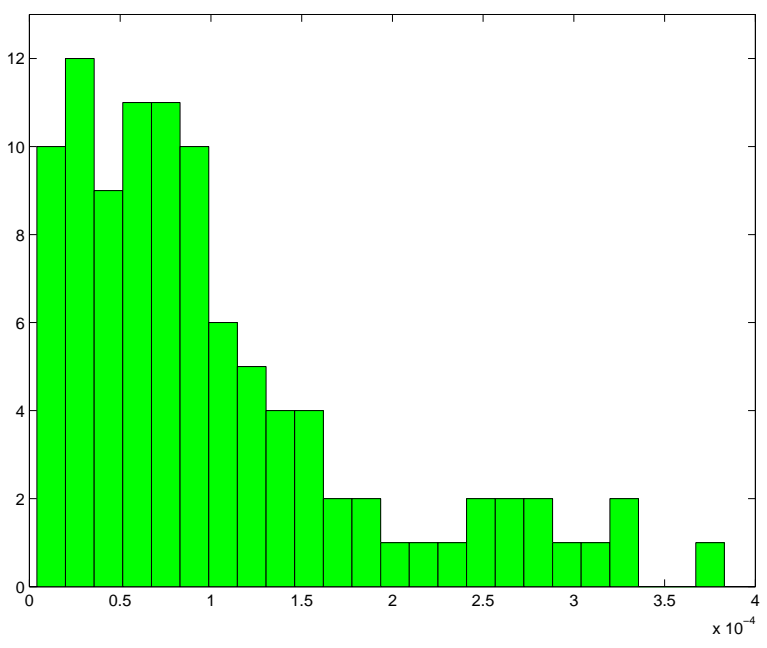

Figure 6A: Uniform: MSE.

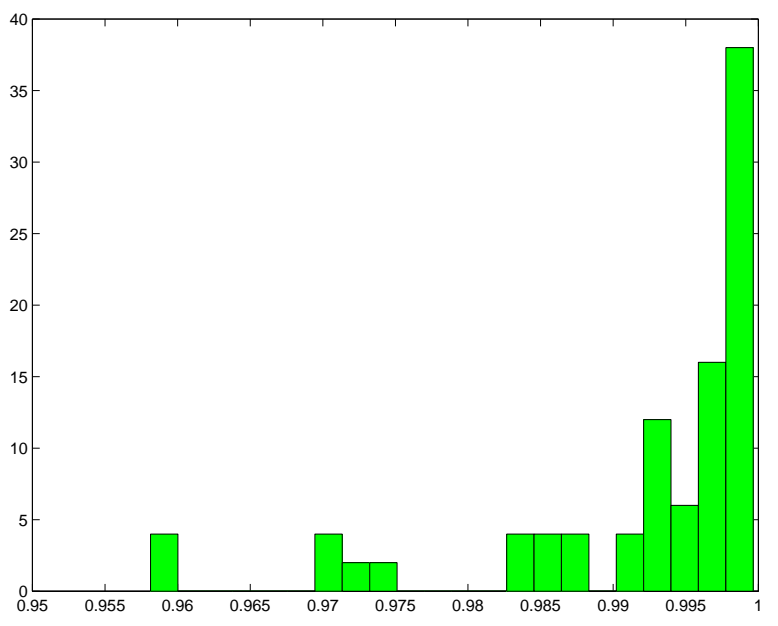

Figure 6C: Uniform: Two-fund separation agent 2.

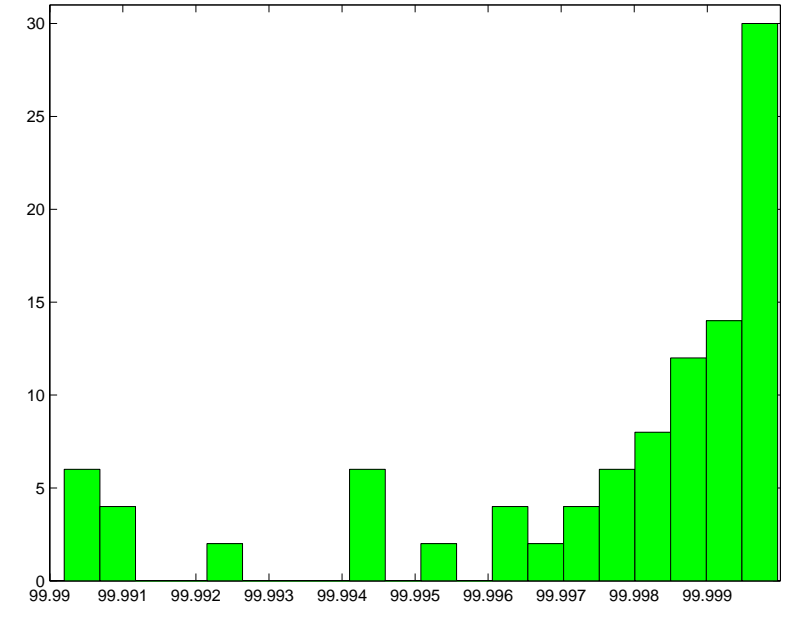

Figure 6B: Uniform: 100 Pricing $R^{2}$.

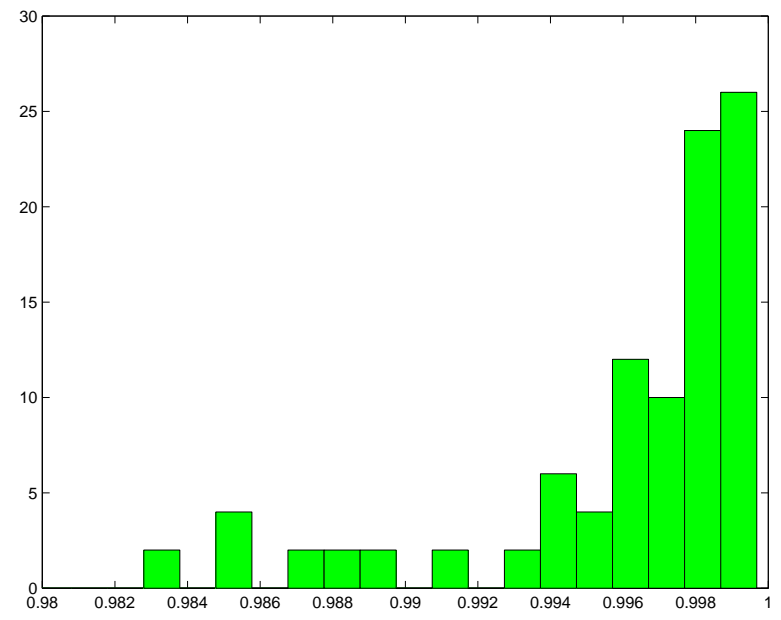

Figure 6D: Uniform: Two-fund separation agent 3 . 
The way to generate $f_{s}^{j}, j=2, \ldots, J$, from a single realization of a normally distributed random variable is the same as in Section 3. The same applies to the other factor.

From Figure 7 we may conclude that the one factor framework is certainly not the driving force that makes CAPM work. Also in the two factor set-up, for a variety of factor loads, with assets that are different in the importance of the idiosyncratic shocks, CAPM turns out to be an excellent model.

\subsection{Options}

Since markets are incomplete the introduction of an option on one of the assets will generally change all equilibrium prices (see Detemple and Selden (1991)). Therefore one might expect that the introduction of an option worsens CAPM-pricing considerably. Furthermore, given the robustness of CAPM in the earlier examples, it is interesting to see if it is possible to give an equilibrium pricing formula for options in incomplete markets via CAPM.

Another reason to introduce an option is that this is an asset with the capacity to seriously alter the higher order moments of an asset portfolio. One possible explanation for our results obtained so far is that asset markets are very incomplete, which makes it difficult for households to change the higher order moments of the returns of their portfolios. Although households care for higher order moments, the mix of marketed assets makes it difficult to affect the higher order moments. With the introduction of an option this clearly changes. Agents have then a possibility to limit downwards risk, which is exactly the kind of risk agents with CRRA utility functions are concerned about, but mean-variance optimizers are not.

In order to investigate this issue more closely we introduce a call option on the most risky asset. Specifically we have a 9 -th security which pays $\max \left(d_{s}^{j}-X, 0\right)$ in state $s$, with $X$ the strike price of the call option.

Suppose we consider the uniquely determined equilibrium pricing vector $\pi_{A}^{*}$ of the economy without the option, and we use this pricing vector to price the option. Given the reasoning of the previous paragraph, at those prices one would expect the call option (in combination with the bond) to be more attractive to the agents than the stock, exactly because of the higher order moments. So the equilibrium price of the call option should be higher than the one computed by CAPM-pricing, in order to make that asset less appealing. As a consequence, the expected equilibrium return of the call option should be less than the one predicted by CAPM.

To examine different options, we draw $X$ out of the uniform distribution for each example. To avoid options that are either too far in or too far out of the money we determine in each example the minimal dividend paid out by asset $8, \underline{d}^{8}=\min _{s=1, \ldots, S} d_{s}^{8}$, and the maximal dividend paid out, $\bar{d}^{8}=\max _{s=1, \ldots, S} d_{s}^{8}$. We then draw $X$ out of a uniform distribution on $\left[0.5 \cdot\left(1.02+\underline{d}^{8}\right), 0.5 \cdot\left(1.02+\bar{d}^{8}\right)\right]$. Note that 1.02 is the expected dividend of asset 8 . The strike price is always between the average of the minimal dividend and the 


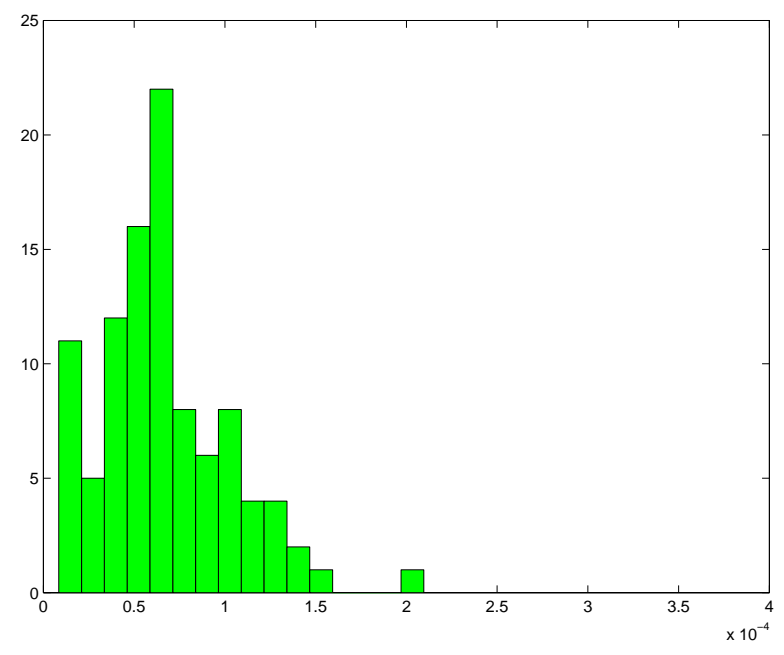

Figure 7A: Two-factor: MSE.

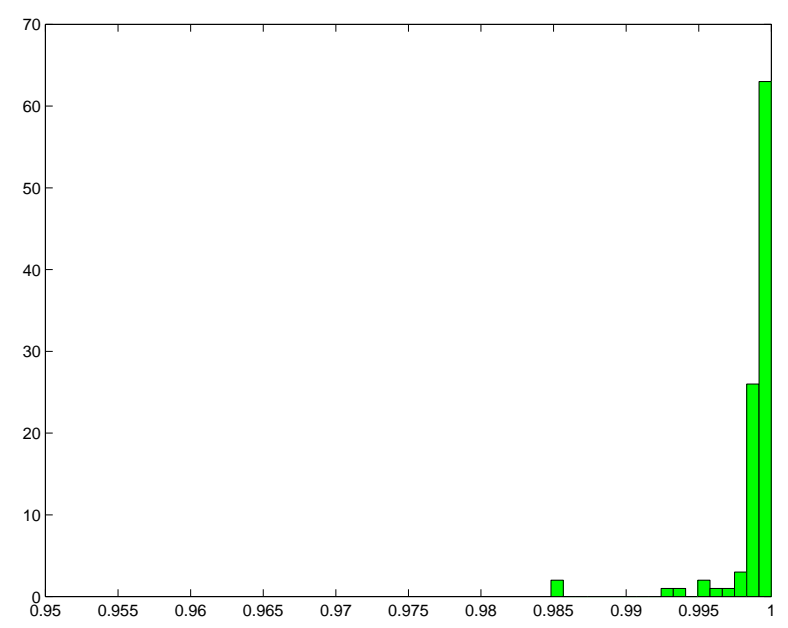

Figure 7C: Two-factor: Two-fund separation agent 2 .

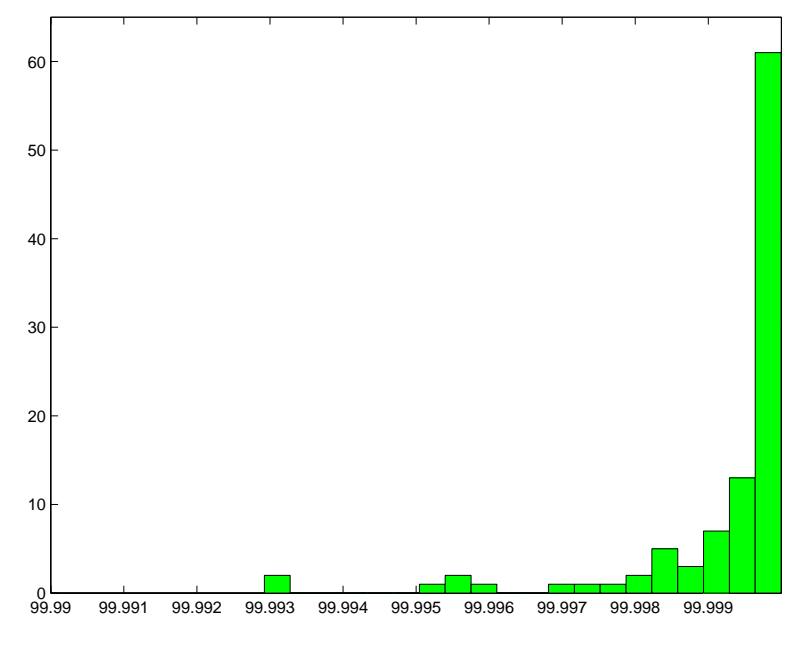

Figure 7B: Two-factor: 100- Pricing $R^{2}$.

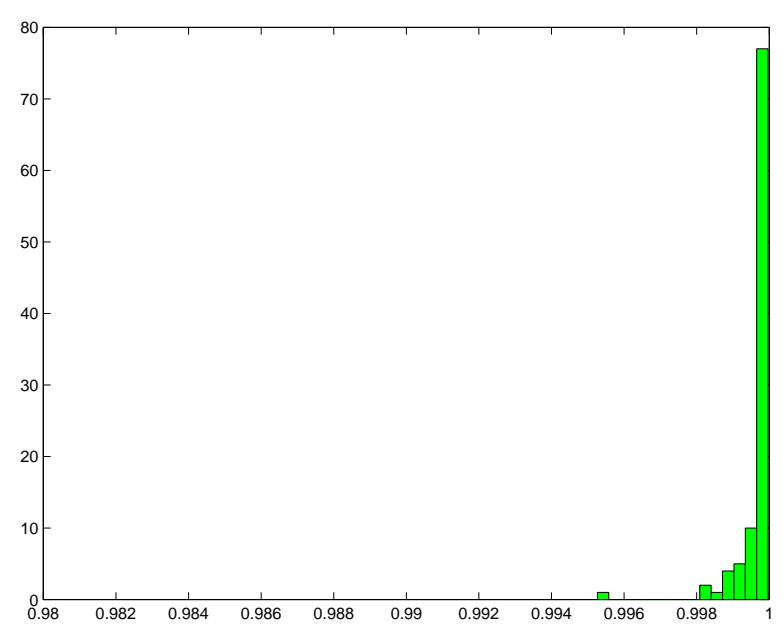

Figure 7D: Two-factor: Two-fund separation agent 3 . 
expected dividend, and the average of the expected dividend and the maximal dividend. The results are given in Figures 8a-d.

The MSE in Figure 8 refers to the MSE of the pricing of the stocks only. The option is analyzed in detail in Figure 9. It turns out that the MSE, and the Two-fund $R^{2}$ are comparable to the ones given before. The Pricing $R^{2}$ is somewhat less good than before, but is still excellent. Surprisingly, we have found no systematic effect of the introduction of the option on the price of asset 8. In some examples the introduction of an option raised the price above the CAPM-prediction, in others it has been lower.

Figure 9 analyzes the pricing of the option by CAPM. According to CAPM, a call option is a very risky asset. It has zero pay-offs in bad states of nature, and very high in good states of nature. The covariance of a call option with the market portfolio is very high, which is also clear from Figure 9, where it is shown that the option's $\beta$ varied from 5 to 35 in the economies generated. Notice that, as we expected, there is indeed an over-prediction of the expected return of an option by CAPM. In all economies generated, CAPM underpriced the call option. The misprediction was relatively small when the option's $\beta$ is low, say below 10, but may get quite severe for call options with a very high strike price, which are the ones with a high $\beta$. Notice, however, that a higher $\beta$ of an option also corresponds to a higher excess return, which makes the relative misprediction less bad. Still, the overprediction of call option returns is more than linearly increasing in an option's $\beta$, whereas the excess return itself is still roughly linear.

It is surprising that the Pricing $R^{2}$ and the MSEs of stocks remained so good in all economies, even when the option was sometimes seriously under-priced by CAPM. In fact, it may even be perceived as an inconsistency that the Pricing $R^{2}$ is virtually exactly correct, and the option is seriously mispriced. Indeed, when CAPM-pricing is highly correlated with $\pi_{A}^{*}$, almost all assets are priced very well. The only exceptions are those like options with a very high strike price. Such an asset pays off in a few (less than 10) states of the 32,768 only. A high correlation with $\pi_{A}^{*}$ is not inconsistent with a fairly different state price in a negligible fraction of states only.

\section{Interpretation AND CONCLUSION}

In order to show that the CAPM-pricing formula holds, one needs strong assumptions either on preferences or on dividends and endowments (see Berk (1996)). However, examining the robustness of CAPM by computing equilibria, we find that CAPM provides an excellent approximation to equilibrium excess returns and portfolio-holdings for a wide variety of preferences, dividends and endowments.

This result is very puzzling for two separate reasons. First, there is no a priori reason why in the absence of mean-variance prefernces or normal returns, the CAPM-pricing formula should provide a good approximation to actual equilibrium prices if there is only one agent in the economy or if markets are complete (which is observationally equivalent). 


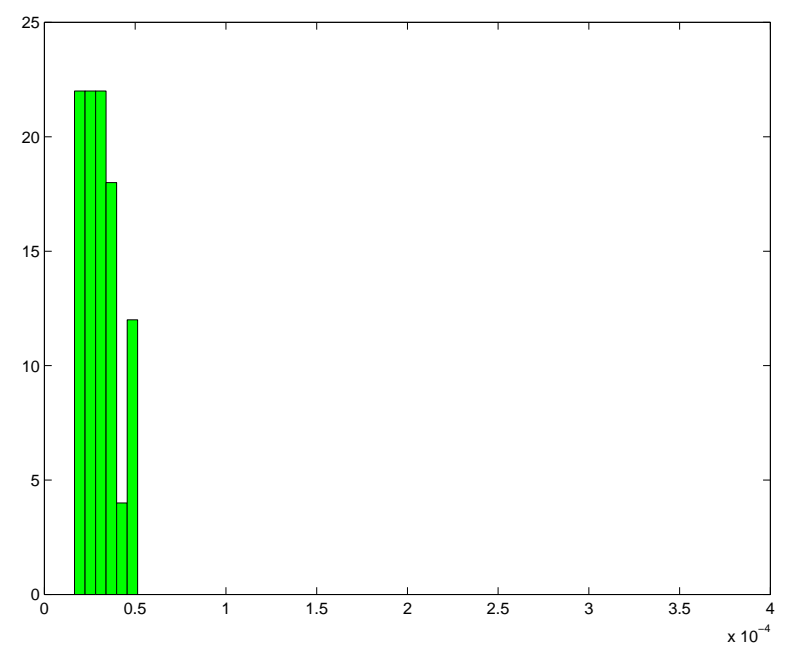

Figure 8A: Option: MSE.

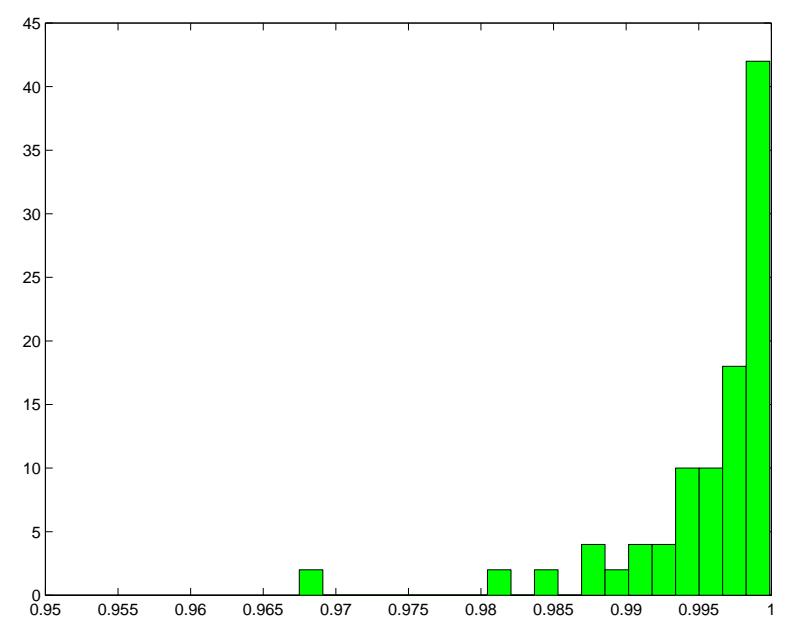

Figure 8C: Option: Two-fund separation agent 2 .

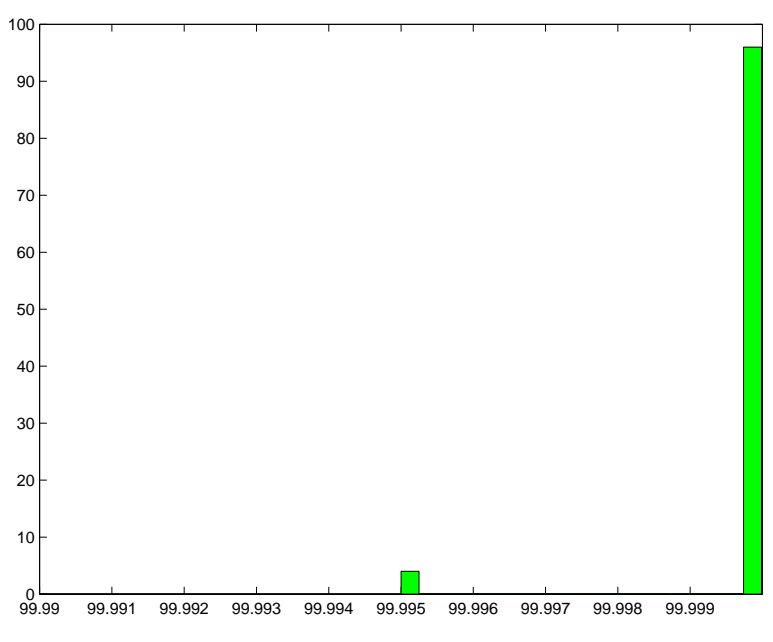

Figure 8B: Option: 100. Pricing $R^{2}$.

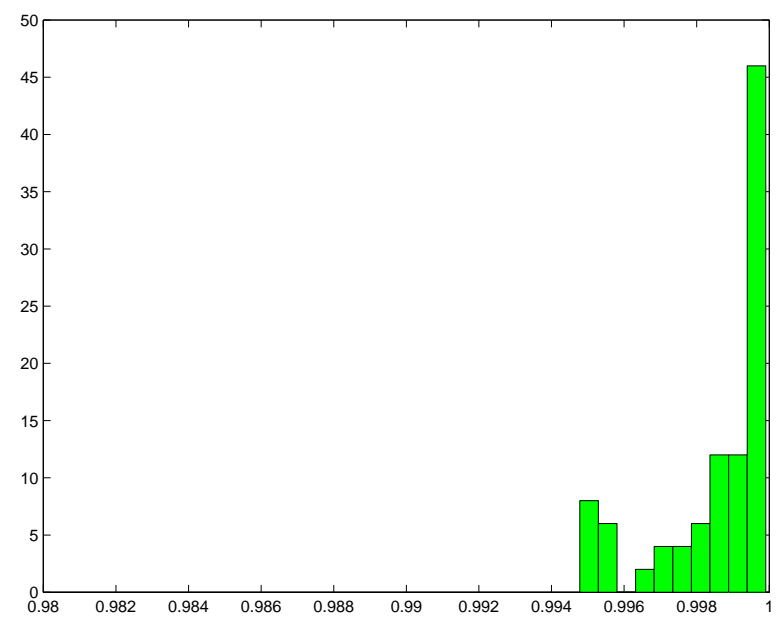

Figure 8D: Option: Two-fund separation agent 3 . 


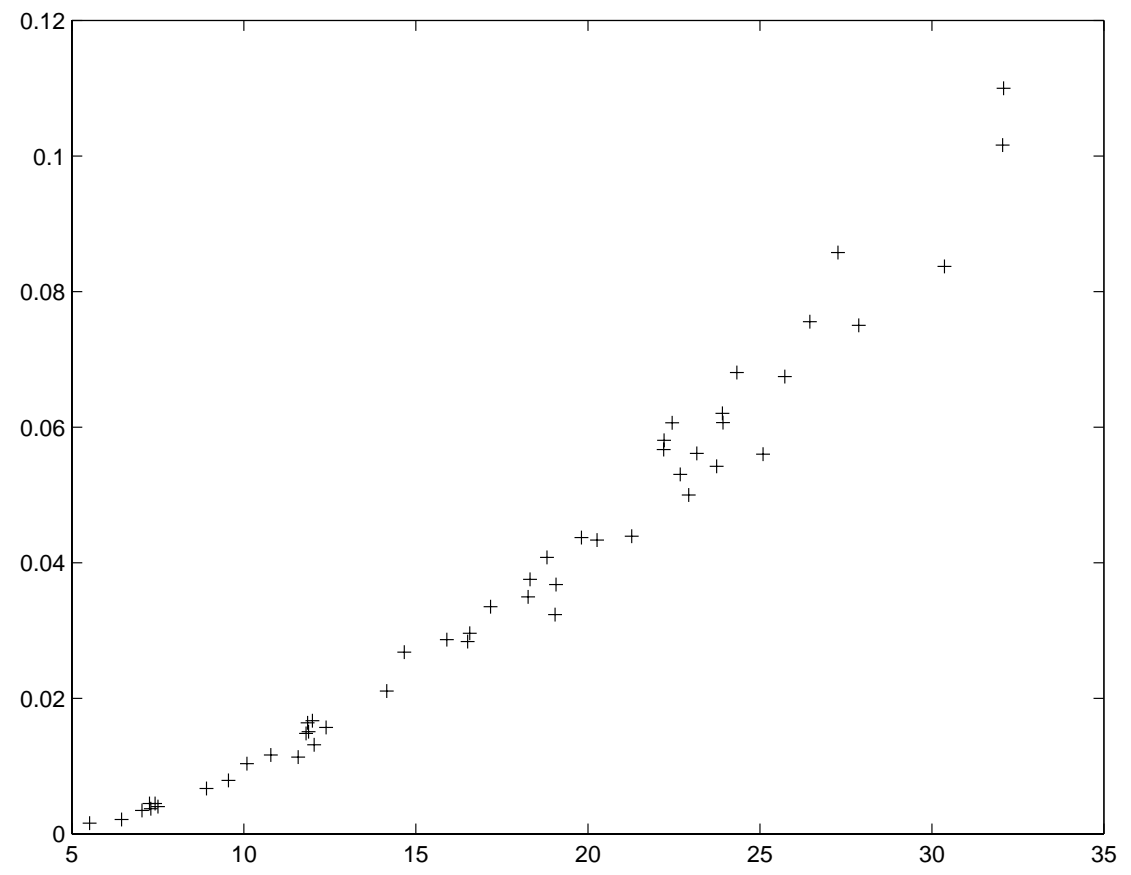

FIgURE 9: Option: over-prediction of return against option's $\beta$. 
Secondly one would expect the presence of heterogeneous agents and incomplete financial markets to alter the pricing implications of consumption based pricing models significantly.

We compute the complete market equilibirum for the examples considered. In almost all of the cases the complete markets pricing errors lie even below the incomplete market errors. In order to understand this first puzzle, consider the simplest possible setup with a single agent who has log-utility and no labor-income. Suppose that the dividends of stocks $j=2, \ldots, 8$ are given by

$$
d_{s}^{j}=\phi_{j}\left(\mu+\varepsilon_{s}^{1}\right)+\varepsilon_{s}^{j},
$$

where all $\varepsilon$ are iid. From Section 2 we know that if we normalize $e_{0}=1$ the pricing errors of the CAPM pricing formula for asset $j$ is given by

$$
\Delta=\mathrm{E} d^{j}-\frac{\mathrm{E}\left(d^{j} / \tilde{e}\right)}{\mathrm{E}(1 / \tilde{e})}-\left(\mathrm{E} \tilde{e}-\frac{1}{\mathrm{E}(1 / \tilde{e})}\right) \frac{\operatorname{cov}\left(d^{j}, \tilde{e}\right)}{\operatorname{var}(\tilde{e})}
$$

The results in this paper might become a little more plausible if one realizes that if factorweights $\left(\phi_{j}\right)_{j=2, \ldots, 8}$ are such that $\sum_{j=2}^{8} \phi_{j}=1$ then $\Delta=0$. Substituting for $d^{j}$ and $\tilde{e}$, since there are 7 stocks and since $\frac{\operatorname{cov}\left(d^{j}, \tilde{e}\right)}{\operatorname{var}(\tilde{e})}=\left(\phi_{j}+1\right) / 8$ we obtain

$$
\Delta=\phi_{j} \mu-\frac{\mathrm{E} \frac{\phi_{j}\left(\mu+\epsilon_{1}\right)+\epsilon_{j}}{\mu+\sum_{i=1}^{8} \epsilon_{i}}}{\mathrm{E} \frac{1}{\mu+\sum_{i=1}^{8} \epsilon_{i}}}-\left(\mu-\frac{1}{\mathrm{E} \frac{1}{\mu+\sum_{i=1}^{8} \epsilon_{i}}}\right)\left(\phi_{j}+1\right) / 8
$$

In order to show that $\Delta=0$ the crucial insight is that

$$
\frac{\mathrm{E} \frac{\epsilon_{j}}{\mu+\sum_{i=1}^{8} \epsilon_{i}}-1 / 8}{\mathrm{E} \frac{1}{\mu+\sum_{i=1}^{8} \epsilon_{i}}}+1 / 8 \mu=0 .
$$

For $\sum_{j=2}^{8} \phi_{j} \neq 1, \Delta \neq 0$ but the resulting pricing errors are small. As long as the coefficient of relative risk aversion remains low the pricing errors remain low if we vary the utility functions (see Figure 3).

However, while this argument shows that given our calibration one would expect CAPM to provide a good approximation to prices if there is only a single agent, one has to compute equilibria in order to assess how well CAPM predicts equilibrium prices in economies with heterogeneous agents and incomplete markets. Our current computational experience suggests that CAPM is an excellent tool to price assets in realistically calibrated economies with incomplete markets. The results seem to be robust for a wide variety of cases. The form of the utility functions, the distribution of the shocks, the number of factors, and the introduction of options do not affect our results. The question which then arises out of the analysis in this paper is why the presence of incomplete markets does not have significant effects on cross-sectional returns. 


\section{References}

[1] Benartzi, S., and R.H. Thaler (1995), "Myopic Loss Aversion and the Equity Premium Puzzle," Quarterly Journal of Economics, 440, 73-92.

[2] Berk, J.B. (1997), "Necessary Conditions for the CAPM," Journal of Economic Theory, 73, 245-257.

[3] Brown, D.J., P.M. Demarzo, and B.C. Eaves (1996), "Computing Equilibria When Asset Markets Are Incomplete," Econometrica, 64, 1-27.

[4] Cass, D., and J.E. Stiglitz (1970), "The Structure of Investor Preferences and Asset Returns, and Separability in Portfolio Allocation: A Contribution to the Pure Theory of Mutual Funds," Journal of Economic Theory, 2, 122-160.

[5] Chamberlain, G. (1983), "A Characterization of the Distributions That Imply MeanVariance Utility Functions," Journal of Economic Theory, 29, 185-201.

[6] Connor, G. (1984), "A Unified Beta Pricing Theory," Journal of Economic Theory, 34, 13-31.

[7] Detemple, J.B., and P. Gottardi (1998), "Aggregation, Efficiency and Mutual Fund Separation in Incomplete Markets," Economic Theory, 11, 443-455.

[8] Detemple, J.B., and L. Selden (1991), "A General Equilibrium Analysis of Option and Stock Market Interactions," International Economic Review, 32, 279-303.

[9] Fama, E.F. and K.R. French (1992), "The Cross-Section of Expected Stock Returns," Journal of Finance, 47, 427-465.

[10] Feldstein, M.S. (1969), "Mean-Variance Analysis in the Theory of Liquidity Preference and Portfolio Selection," Review of Economic Studies, 36, 5-12.

[11] Garcia, C. and W. Zangwill (1981), Pathways to Solutions, Fixed Points, and Equilibria, Prentice Hall, Englewood Cliffs.

[12] Geanakoplos, J.D., and H.M. Polemarchakis (1986), "Existence, Regularity, and Constrained Suboptimality of Competitive Allocations when the Asset Market is Incomplete," in W.P. Heller, R.M. Starr, and D.A. Starrett (eds.), Uncertainty, Information and Communication: Essays in Honor of K.J. Arrow, Vol. III, Cambridge University Press, Cambridge, 65-96.

[13] Geanakoplos, J., and M. Shubik (1990), "The Capital Asset Pricing Model as a General Equilibrium With Incomplete Markets," The Geneva Papers on Risk and Insurance, $15,55-72$. 
[14] Gottardi, P., and T. Hens (1996), "The Survival Assumption and Existence of Competitive Equilibria when Asset Markets are Incomplete," Journal of Economic Theory, 71, 313-323.

[15] Hens, T. (1991), Structure of General Equilibrium Models with Incomplete Markets, Doctoral Dissertation, University of Bonn.

[16] Herings, P.J.J. and F. Kubler (2000), "Computing Equilibria in the Finance Version of the General Equilibrium Model with Incomplete Asset Markets," working paper.

[17] Judd, K.L. (1997), "Computational economics and economic theory: Subsitutes or complements ?" Journal of Economic Dynamics and Control, 21, 907-942.

[18] Lintner, J. (1965), "The Valuation of Risky Assets and the Selection of Risky Investments in Stock Portfolios and Capital Budgets," Review of Economics and Statistics, 47, 13-37.

[19] Magill, M.J.M., and M. Quinzii (1996), Theory of Incomplete Markets, MIT Press, Cambridge.

[20] Markowitz, H.M. (1959), Portfolio Selection: Efficient Diversification of Investment, Yale University Press, New Haven.

[21] Mehra, R., and E.C. Prescott (1985), "The Equity Premium, A Puzzle," Journal of Monetary Economics, 15, 145-161.

[22] Oh, G. (1996), "Some Results in the CAPM with Nontraded Endowments," Management Science, 42, 286-293.

[23] Owen, R., and R. Rabinovitch (1983), "On the Class of Elliptical Distributions and Their Applications to the Theory of Portfolio Choice," Journal of Finance, 38, 745752 .

[24] Ross, S.A. (1978), "Mutual Fund Separation in Financial Theory: The separating Distributions," Journal of Economic Theory, 17, 254-286

[25] Schmedders, K. (1998), "Computing Equilibria in the General Equilibrium Model with Incomplete Asset Markets," Journal of Economic Dynamics and Control, 22, 1375-1401.

[26] Sharpe, W.F. (1964), "Capital Asset Prices: A Theory of Market Equilibrium under Conditions of Risk," Journal of Finance, 19, 425-442.

[27] Tobin, J. (1958), "Liquidity Preference as Behavior toward Risk," Review of Economic Studies, 25, 65-86. 
[28] Willen, P.T. (1997), A Model which Allows for Comparison of Different Levels of Market Incompleteness, mimeo, Yale University, New Haven. 